\title{
Efficacy and safety of Panax notoginseng saponin therapy for acute intracerebral hemorrhage, meta-analysis, and mini review of potential mechanisms of action
}

\author{
Dongying $X u^{1}{ }^{*}$, Ping Huang ${ }^{1}$, Zhaosheng $\mathrm{Yu}^{2}$, Daniel H. Xing ${ }^{3}$, Shuai Ouyang ${ }^{4}$ and Guoqiang Xing ${ }^{*}$ \\ ${ }^{1}$ Faculty of Nursing, Guangxi University of Chinese Medicine, Nanning, China \\ 2 Department of Oncology, Huanggang Hospital of Traditional Chinese Medicine, Huanggang, China \\ ${ }^{3}$ Thomas Wootton High School, Rockville, MD, USA \\ ${ }^{4}$ School of Business, University of Alberta, Edmonton, $A B$, Canada \\ ${ }^{5}$ Lotus Biotech.com LLC, Rockville, MD, USA
}

\section{Edited by:}

Yumin Zhang, Uniformed Services University of the Health Sciences, USA

\section{Reviewed by:}

Stefan Plantman, Karolinska

Institutet, Sweden

Zhengtao Wang, Shanghai University

of Traditional Chinese Medicine, China

*Correspondence:

Dongying Xu, Faculty of Nursing, Guangxi University of Chinese

Medicine, 61 Dongge Road, Nanning 530022, Guangxi, China

e-mail:donnaxu_cn@hotmail.com; Guoqiang Xing, Lotus Biotech.com LLC, John Hopkins University-MCC, 9601 Medical Center Drive, Suite 227, Rockville, MD 20850, USA

e-mail: gxing99@yahoo.com
Intracranial/intracerebral hemorrhage $(\mathrm{ICH})$ is a leading cause of death and disability in people with traumatic brain injury (TBI) and stroke. No proven drug is available for $\mathrm{ICH}$. Panax notoginseng (total saponin extraction, PNS) is one of the most valuable herb medicines for stroke and cerebralvascular disorders in China. We searched for randomized controlled clinical trials (RCTs) involving PNS injection to treat cerebral hemorrhage for meta-analysis from various databases including the Chinese Stroke Trials Register, the trials register of the Cochrane Complementary Medicine Field, the Cochrane Central Register of Controlled Trials, MEDLINE, Chinese BioMedical disk, and China Doctorate/Master Dissertations Databases. The quality of the eligible trials was assessed by Jadad's scale. Twenty (20) of the 24 identified randomized controlled trials matched the inclusive criteria including 984 $\mathrm{ICH}$ patients with PNS injection and $907 \mathrm{ICH}$ patients with current treatment (CT). Compared to the CT groups, PNS-treated patients showed better outcomes in the effectiveness rate (ER), neurological deficit score, intracranial hematoma volume, intracerebral edema volume, Barthel index, the number of patients died, and incidence of adverse events. Conclusion: PNS injection is superior to $\mathrm{CT}$ for acute $\mathrm{ICH}$. A review of the literature shows that PNS may exert multiple protective mechanisms against ICH-induced brain damage including hemostasis, anti-coagulation, anti-thromboembolism, cerebral vasodilation, invigorated blood dynamics, anti-inflammation, antioxidation, and anti-hyperglycemic effects. Since vitamin $\mathrm{C}$ and other brain cell activators (BCA) that are not considered common practice were also used as parts of the CT in several trials, potential PNS and BCA interactions could exist that may have made the effect of PNS therapy less or more impressive than by PNS therapy alone. Future PNS trials with and without the inclusion of such controversial BCAs as part of the CT could clarify the situation. As PNS has a long clinical track record in Asia, it could potentially become a therapy option to treat ICH in the US and Europe. Further clinical trials with better experimental design could determine the long-term effects of PNS treatment for TBI and stroke.

Keywords: notoginsenosides, botanical medicine, nutraceuticals, TBI and stroke recovery, randomized controlled clinical trials, hemostasis, anti-coagulation, pharmacological mechanisms

\section{INTRODUCTION}

Traumatic brain injury (TBI) is a leading cause of death and disability in young people (1). Every year approximately 1.5 million people die and at least 10 million people are hospitalized after TBI (2). The incidence of TBI fatality and disability rates are higher in developing countries than in developed countries (3).

Secondary brain damage due to continued intracranial and intracerebral bleeding and hemorrhage swelling is a common cause of morbidity and mortality $(4,5)$. In one clinical trial, $56 \%$ of the patients with mild, moderate and severe TBI developed intracranial hemorrhage (6). Another study showed that $51 \%$ of TBI patients developed progressive intracranial/intracerebral hemorrhage ( $\mathrm{ICH})$, and hemorrhage expansion during the first 24-48 h after hospital admission (7). Prognostic studies have shown that ICH is associated with increased mortality and disability 6 months after injury $(8,9)$. One recent survey reported that TBI patients who developed ICH showed a 10 -fold increase in stroke incidence 3 months after the injury when compared to TBI patients without ICH (10).

Acute intracerebral hemorrhage $(\mathrm{AICH})$ accounts for only about $10 \%$ of the people with stroke, and is the most lethal form of stroke compared to the ischemic stroke. Thus, ICH is among the most devastating disorders and a leading cause of disability and mortality of people with severe stroke, hypertension, 
and TBI (11). During the last decade, the incidence of ICH has increased steadily in Asian countries (12) and it accounts for 20\% and $\sim 10 \%$ of strokes in low-middle and high income countries, respectively (13).

So far, few proven therapies exist for ICH. Hematoma expansion, perihematomal edema with increased intracranial pressure, intraventricular extension of hemorrhage with hydrocephalus, seizures, venous thrombotic events, hyperglycemia, increased blood pressure, fever, and infections are among the complications of ICH as recently reviewed by Balami and Buchan (14). Current treatment of $\mathrm{ICH}$ is supportive and life-sustaining rather than a complete cure that aims to limit secondary brain damage and associated complications $(15,16)$. Considering the very limited therapeutic options for patients with $\mathrm{ICH}$, recent studies suggest that evidence-based alternative and complimentary medicines could be effective in reducing the adverse effects early in the course of ICH and in improving its prognosis as found in the treatment of cerebral ischemia (17).

Panax notoginseng [(Burk.) F.H. Chen] (also called Sanqi in Chinese), is one of the most valuable Chinese herbal medicine. $P$. notoginseng is a perennial plant, mainly grown in the high mountain areas of Southwest China. Its roots are harvested after 3-5 years of growth (Figure 1). P. notoginseng has numerous hematological and pharmacological effects, which include regulation of platelet aggregation and platelet free calcium levels, reducing blood viscosity, improving local blood supply and circulation to end stasis, cerebral vasodilation, analgesic, hypolipidemic, hemostatic, anti-edema, anti-hyperglycemia, antioxidation, anti-inflammation, and anti-apoptosis (18, 19).
P. notoginseng saponins extract (PNS) ameliorate learning and memory deficits in animals (20-26), probably by inhibiting oxidative stress and apoptosis and by stimulating neurogenesis $(21,22$, 27-33). PNS is effective against ICH, transient focal ischemia, and cerebral infarction probably in part through improved brain blood circulation and energy metabolism (34-38). P. notoginseng has been used alone and as a key tonic ingredient in many other patent Chinese medicine for treatment of a variety of health conditions and has been proved to be effective in animal models of cerebral ischemia/reperfusion injury, arterial thrombosis, cardiovascular disorders, Alzheimer disease, diabetes and obesity, erectile dysfunction, neurodegeneration, neuroinflammation, oxidative stress, neurotoxicity, organ injury, and cancer (38-47).

The ability of notoginseng to normalize hemorheological parameters is due to the presence of multiple active compounds including different ginsenosides and notoginsenosides, some of which appear to have similar yet differential effects. Ginsenosides $\operatorname{Rg} 1$ and Rb1, and notoginsenoside R1 are the main active ingredients present in high concentrations in PNS, which contains more than 20 different ginsenosides and notoginsenosides (4855). Purified and patented PNS under different trade names, i.e., Xuesaitong (51, 56-59), Xueshuantong (60), and Lulutong have been approved for treatment of stroke and other cerebral disorders in China. Intravenous injection of PNS has been developed for critical care because orally administered PNS has a low permeability, poor intestinal absorption, and bioavailability $(51,61,62)$ due to the relative large sugar molecular mass (>500 Da), high molecular flexibility, high hydrogen binding capacity, and low lipophilicity.
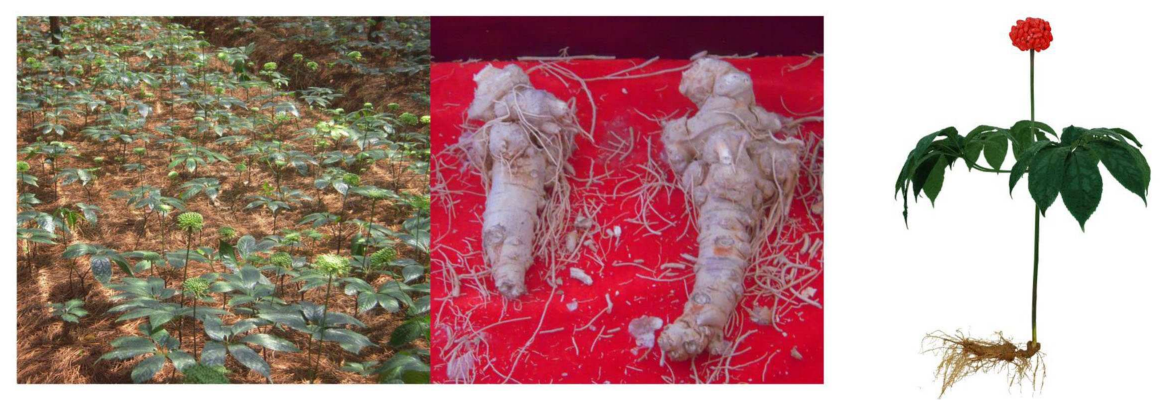

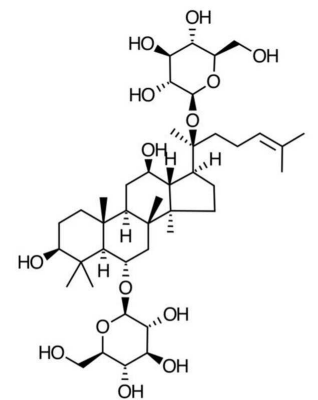

Rg1

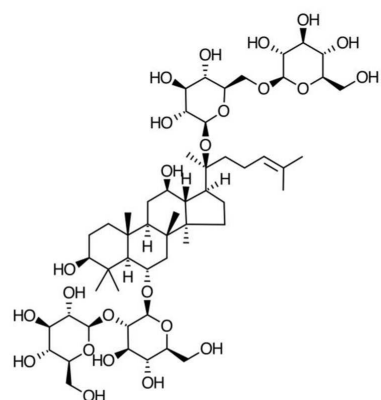

Rb1

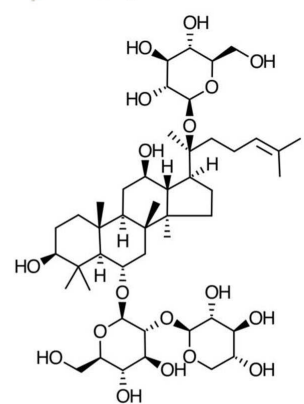

R1

FIGURE 1 | Panax notoginseng (P. notoginseng, Sanqi): clockwise from top left: in cultivation, the roots, artistic drawing a Sanqi plant, the main chemical ingredients of PNC: notoginsenoside R1, ginsenosides Rb1, and Rg1. 
In vivo studies showed marked variability of $\mathrm{Rb} 1$ bioavailability among different administration routes to rats: i.v. (intravenous) $(100 \%)>$ p.v. (portal venous) $(59.49 \%)>$ i.d. (intra-duodenal) $(2.46 \%)>$ p.o. (peroral) (0.64\%) (63). After absorption, PNS has a long residual time, but individual ginsenosides and notoginsenosides vary in their elimination rate. For example, the half-life is much longer and peak concentration higher for ginsenoside $\mathrm{Rb} 1$ than that for ginsenoside $\mathrm{Rg} 1$ and notoginsenoside $\mathrm{R} 1$, due to the slower clearance and longer residence time of Rb1 (61, 63-67). In fact, ginsenoside $\mathrm{Rb} 1$ is considered a pharmacokinetic marker of PNS (67).

Multiple clinical studies have been conducted in China in recent years to explore the use of PNS injection in the treatment of cerebral hemorrhage and ICH. These studies have yet to be systematically evaluated for the efficacy and safety to provide evidence and guidance for further clinical application of PNS for cerebral hemorrhage. Because many of these trials were reported in un-indexed Chinese medical journals, the international research community may not have access to these findings. In this study, we reviewed randomized controlled clinical trials (RCTs) published in Chinese journals that involved PNS injection treatment for ICH. The results suggest that PNS injection has a wide range of different protective mechanisms and is a better treatment than current treatments for ICH.

\section{MATERIALS AND METHODS INCLUSIVE AND EXCLUSIVE CRITERIA}

Only RCTs that included a comparison of the efficacy and safety of $P$. notoginseng saponin (PNS) injection treatment with that of current treatment (CT) in patients with $\mathrm{AICH}$ resulted from hypertension or wind stroke were included. The diagnostic criteria of acute $\mathrm{ICH}$ in trials were in accordance with the criteria of diagnosis of various types of cerebrovascular disease updated at the 4th Annual Conferences of Chinese Society of Neurology (68): subjects should not suffer from secondary AICH or other diseases such as hematologic diseases, intracranial aneurysms, intracranial tumors, cerebral arteries, venous malformations, severe comas, and cardiac, hepatic, or renal diseases. There was no restriction on race, but the majority of patients were thought to be of the Chinese Han ethnic group. The PNS intervention is defined as intravenous drip of the commercially available PNS preparations that are approved by Chinese Food and Drug Administration (CFDA) for clinical use alone, or in combination with other routine therapies for subjects in the treatment groups. The control $\mathrm{ICH}$ subjects received current treatment other than PNS treatment. The outcome measures included the effectiveness rate (ER), neurological deficit score (NDS), intracerebral hematoma volume (IHV), intracerebral edema volume (IEV), Barthel index (BI), and number of patients died (NDP), as well as incidence of adverse events after treatments with PNS or CT, respectively. The BI is an interviewer-based disability profile scale developed by D.W. Barthel in 1965 to assess physical functions, specifically self-care abilities and ambulation (e.g., stair climbing) in 10 areas, including bowel and bladder control. The patient is scored from 0 to 15 points in various categories, depending on his or her need for help, such as in feeding, bathing, dressing, and walking.

\section{SEARCH STRATEGY}

The search strategy was developed by modifying the reported strategies used for herbal medicines in a Cochrane review (69). We retrieved the literatures of relevant clinical trials by electronic searching and by hand searching, regardless of language or publication status. Many electronic databases were searched, including the Chinese Stroke Trials Register, the trials register of the Cochrane Complementary Medicine Field, the Cochrane Central Register of Controlled Trials, MEDLINE, CINAHL, AMED, Chinese BioMedical disk, Wanfang Chinese Scientific Journal Database, VIP, China National Knowledge Infrastructure, Traditional Chinese Medicine Database, Chinese Medical Current Contents, China Doctorate/Master Dissertations Full-Text Databases, and China Proceedings of Conference Databases. The reference lists of retrieved papers were further scanned for any possible titles matching the inclusive criteria. A hand search with an emphasis on relevant journals pertaining to stroke, senile disease, neurology, complementary, and alternative medicine was carried out to explore entities matching the inclusive criteria among periodicals, journals, and symposium abstracts found in libraries of Guangxi Chinese Medical University (date of last search: December, 2013).

\section{DATA EXTRACTION}

Full-text articles of each potential eligible trial were retrieved and assessed by two independent reviewers (Dongying Xu and Ping Huang) to determine if the articles should be recruited and further analyzed according to the inclusive and exclusive criteria. Missing information was sought by contacting the article authors. A data abstraction form was used to summarize key information from included trials, and key information was extracted by one reviewer and confirmed by the other. Any disagreements were resolved by discussion.

\section{DATA ANALYSIS}

The meta-analysis was carried out by using Revman 5.3 software (Cochrane Collaboration) to combine and analyze the data from the individual trials. The statistical validity of combining various trials was assessed by examining the homogeneity of outcomes from trials using a Q-test (Mantel-Haenszel Chi-square test). The results of the combined trials were calculated with random or fixed-effect models. The measurements of each category's data were evaluated by a weighted mean difference (WMD) or odd ratio (OR), and by $95 \%$ confidence intervals ( $95 \% \mathrm{CI}$ ). The methodological quality of all included trials was assessed by Jadad's scale that evaluated randomization, double blinding, and dropout rate of the trials by ranking them with $1-5$ points. The trials that scored with 1 or 2 points were considered low-quality trials, while those that scored with $3-5$ points were considered high-quality trials (70).

\section{RESULTS \\ EXCLUDED AND INCLUDED TRIAL}

The literature search yielded a total of 24 RCTs conducted in China that treated acute intracerebral hemorrhagic patients with intravenous drip of PNS. However, four of these trials were excluded as they did not match the inclusive criteria. Specifically, they are (1) no-comparison made between PNS and RT in trials (two trials) 
and (2) no or unclear measurement report in trials (two trials). Therefore, only 20 trials published in Chinese medical journals, with 1,891 ICH patients that met the study criteria, were included for analysis (71-90).

The characteristics of the patients are shown in Table 1. The trials' size varied from 24 to 200 participants, with an average of 46 patients per trial. All of the patients are adults ranged from 24 to 92 years old with more males than females included (62\% males vs. $38 \%$ females). Hemorrhage/ICH duration was reported in 13 trials, ranging from $4 \mathrm{~h}$ to 20 days. The main causes of intracerebral hemorrhage were wind stroke (15 trials) and hypertension (2 trials) (Table 1).

The classification of the patients (sites of bleeding) is shown in Table 2. Of these, $984 \mathrm{ICH}$ patients received PNS injection treatments that lasted between 10 and 70 days, and 907 patients received current treatments (Table 2 ). Of the PNS injection treatments, 6 trials used Xuesaitong PNS freeze-dry powder injections $(72,73,77-79,85), 7$ trials used Xuesaitong injections $(74,75,84$, 86-88, 90), 5 trials used Xueshuantong injections $(76,80,81,83$, 89), one trial used Lulutong injections (71), and one trial used Sanqi Zaogan injection powder (82). For the CT control groups, dehydration, control of intracranial pressure, anti-hypertensive treatment, symptomatic treatment, neurotrophy medicine, and brain cell activators (BCA) were used. Mannitol, glycerol, and/or ructose injection was used for dehydration (Table 2).

\section{OUTCOME MEASUREMENT}

For evaluating the therapeutic and adverse effects of the PNS treatment and the control groups, the outcome assessment of this study was focused on the ER, NDS, intracerebral hematoma volume (IHV), IEV, BI, and the NDP. The incidence of adverse events after treatments was also evaluated. Thirteen trials reported the number of improved patients. Six trials reported NDS. Eight trials reported intracerebral hematoma volume. Three trials reported intracranial edema volume. Two trials reported BI, and six trials reported the NDP.

\section{META-ANALYSIS ON PNS EFFICACY Sites of bleeding}

There were 15 trials involving 687 ICH patients in the PNS group and 583 patients in the CT group (total of 1,270 ICH patients) that provided detailed descriptions of the bleeding sites including (unilateral and/or bilateral) the basal ganglia, external capsule, internal capsule, frontal lobe, medial occipital lobe, arietal lobe, cerebellum, brainstem, ventricles, and supratentorial hemorrhage (Table 3). Among them, the basal ganglia region was the most common site of hemorrhage, accounting for $45.85 \%(315 / 687)$ of patients in the PNS group, and $41.51 \%(242 / 583)$ of patients in the CT group.

\section{Effectiveness rate}

By using the fixed-effect model, Figure 2 shows the results of metaanalyses on the ER, comparing the therapeutic effect of PNS injection with that of the CT. A total of 13 trials reported the effect rate, which was categorized into three subgroups by the evaluating time: (1) 7 trials assessed the ER at the end of 2 weeks of treatment, (2) 2 trials assessed the rate at the end of 3 weeks, and (3) 5 trials assessed the rate at the end of 4 weeks. There was no significant heterogeneity among these three subgroups $(P=0.47)$. The total overall effect showed significant statistical difference in ER between the PNS and CT groups $(\mathrm{OR}=2.70 ; 95 \% \mathrm{CI}=2.16,3.38 ; P<0.00001)$. There were significant differences in ER between PNS and CT groups assessed at 2 weeks $(\mathrm{OR}=2.73 ; 95 \% \mathrm{CI}=1.92,3.88 ; P<0.00001)$, 3 weeks $(\mathrm{OR}=2.43 ; 95 \% \mathrm{CI}=1.54,3.83 ; P=0.0001)$, and 4 weeks $(\mathrm{OR}=2.87 ; 95 \% \mathrm{CI}=1.97,4.18 ; P<0.00001)$ after the start of treatment. No significant heterogeneity was presented in the analyses of the data $(P=0.55,0.61$, and 0.13$)$ in the three subgroups, respectively. Thus, ICH patients with PNS treatment showed a better therapeutic ER than those in the CT group.

\section{Neurological deficit score}

Neurological deficit score is an important index for the diagnosis of symptom severity and functional recovery of the patients (91). Six of the selected trials reported NDS in this study, with three of them showing NDS $(74,77,86)$ at $7,15,21,28$, and 30 days after PNS treatment (Figure 3). No significant differences in NDS were found between the PNS and CT groups at 7 (77) and 15 days (86). The results of 21 days are controversial, since one trial showed no difference between the PNS and CT groups (74), whereas the other showed a better NDS in the PNS group than in the CT group (77). The NDS was significantly lower in PNS group than that in CT group at 28 days (86) and 40 days (74) after the treatment $(P<0.05, P<0.01$, respectively). No heterogeneity $(P=0.48)$ was found in the NDS. When all data were combined, the results showed significantly reduced NDS in PNS-treated ICH patients than in $\mathrm{ICH}$ patients of the $\mathrm{CT}$ group $(\mathrm{MD}=4.36 ; 95 \%$ $\mathrm{CI}=3.07,5.65 ; P<0.00001)$ (Figure 3).

\section{Intracerebral hematoma volume}

Of the 8 trials that reported IHV, 6 trials showed no differences in IHV between the PNS and CT groups after 4-7 days of treatment $(82,84,85,88,89)(\mathrm{MD}=-0.37 ; 95 \% \mathrm{CI}=-1.60,0.87$; $P=0.58)$ (Figure 4). Three trials $(82,84,87)$ showed significant improvement in IHV in the PNS group (about $25 \%$ less IHV) than in the CT group at 10-14 days after the treatment $(\mathrm{MD}=-3.80$; $95 \% \mathrm{CI}=-5.87,-1.74 ; P=0.0003)$. Four trials showed significantly smaller IHV values in the PNS group (about $40 \%$ less) than in the CT group at 20-21 days after the treatment $(74,81,84$, 89) $(\mathrm{MD}=-4.82 ; 95 \% \mathrm{CI}=-8.32,-1.33 ; P=0.007)$. Another 4 trials showed significantly smaller IHV values in the PNS group (about 50\% less) than in the CT group at 28-40 days after the treatment $(74,82,85,88)(\mathrm{MD}=-5.15 ; 95 \% \mathrm{CI}=-5.98,-4.33$; $P<0.00001)$. These results show a time-dependent effect of PNS treatment on IHC, i.e., significant improvement in IHV occurs after 21 days or more of PNS treatment, but not in the 1st week of treatment $(10-14$ days, $\mathrm{MD}=-3.8 ; 20-21$ days, $\mathrm{MD}=-4.82$; 28-40 days, $\mathrm{MD}=-5.15)$.

\section{Intracerebral edema volume}

Figure 5 shows the results of meta-analyses on IEV. Only three trials $(82,85,89)$ assessed IEV in ICH patients with PNS/CT treatment 7 days after ICH onset. Two of the trials with a duration of 3-4 weeks showed significant differences in IEV between the PNS and CT groups $(65,68)$. The other trial showed no significant difference between the two groups after 14 days of PNS injection due 
Table 1 | Baseline characteristics of each trial used in the meta-analysis.

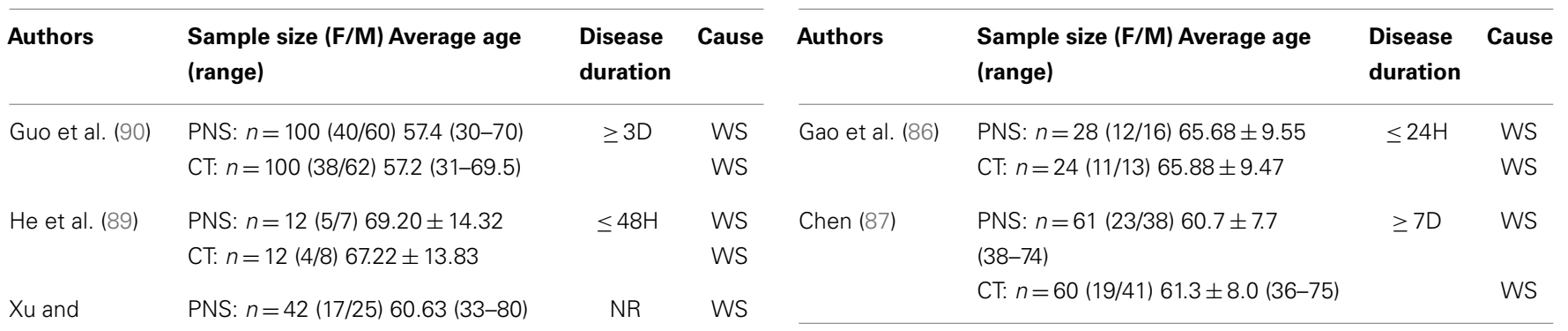

Dong (72) RT: $n=40$ (17/23) 58.30 (34-79)

Li et al. (88)

$$
\begin{aligned}
& \text { A:PNS: } n=60(25 / 35)(24-78) \\
& \text { B:PNS: } n=55(22 / 33)(31-75) \\
& \text { C:CT: } n=60(26 / 34)(40-82)
\end{aligned}
$$

Li and

Yang (73)

PNS: $n=48(13 / 35) 57.2 \pm 9.6$

(45-75)

CT: $n=44(12 / 32) 56.8 \pm 9.4(43-74)$

Tian et al. (74)

PNS: $n=36(16 / 20) 60.32 \pm 5.14$

CT: $n=30(16 / 14) 58.41 \pm 6.33$

Li and Sun (75)

PNS: $n=29(9 / 20) 58.5 \pm 10.8$

(31-82)

CT: $n=31(9 / 22) 57.5 \pm 11.2(33-81)$

Xie et al. (76)

PNS: $n=24$ (6/18) 61.1 (34-89)

CT: $n=22$ (6/16) $61.2(35-92)$

Chen et al. (77) PNS: $n=22$ (7/15) N/A

CT: $n=21(7 / 14) \mathrm{N} / \mathrm{A}$

Dong and

Wang (78)

Zhang

et al. (71)

Zhou et al. (79)

PNS: $n=40(16 / 24) 60.63(33-80)$

CT: $n=38(16 / 22) 58.3(34-79)$

PNS: $n=65$ (N/A) 57.6 (35-69)

CT: $n=65$ (N/A) $58.2(36-70)$

PNS: $n=70(24 / 46) 56.8 \pm 10.4$

(35-78)

CT: $n=70(27 / 43) 55.6 \pm 10.1$

(33-79)

Zheng (80)

PNS: $n=22$ (N/A) N/A

CT: $n=19$ (N/A) N/A

Tang et al. (81) PNS: $n=63(25 / 38) 62.2 \pm 14.6$

CT: $n=63(23 / 40) 62.5 \pm 15.2$

Song (82)

PNS: $n=39(12 / 27) \mathrm{N} / \mathrm{A}$

CT: $n=39(15 / 24)$ N/A

Ding et al. (83) PNS: $n=15$ (6/9) 55.1 (35-74)

CT: $n=17(4 / 13) 57.7(44-72)$

Yuan et al. (84) PNS: $n=67$ (26/41) 59.2 (39-75)

CT: $n=66(27 / 39) 61.3(41-75)$

Ding and

Geng (85)

PNS: $n=86(34 / 52) 60.2 \pm 8.5$

CT: $n=86(37 / 49) 61.4 \pm 8.2$
WS WS

$>20 \mathrm{D}$

WS

CT, current treatment; $D$, day; $H$, hour; HYTN, hypertension; N/A, not applicable; NR, not reported; PNS, Panax notoginseng saponin; WS, wind stroke IStroke including hemorrhagic cerebrovascular accident (CVA), ischemic infarction CVA and subarachnoid hemorrhage, etc. is often collectively referred to as wind stroke in traditional Chinese medicine because of its sudden and acute onset, sudden loss of consciousness with unilateral weakness, numbness, paralysis and dysphasia with or without experiencing unconsciousness, multiple symptoms and rapid alterations in manifestations that are similar to the natural characteristics of wind, which is changing rapidly].

to a small sample size $(89)(\mathrm{MD}=14.87 ; 95 \% \mathrm{CI}=-0.37,30.11)$. Analysis of the combined data showed significant statistical difference in IEV values between the PNS and CT groups $(\mathrm{MD}=10.78$; 95\% CI $=9.07,12.49 ; P<0.00001$ ) (Figure 5). On average, PNS treatment reduced IEV value by about $50 \%$.

NR $\quad$ NR NR

\section{Barthel index}

The BI is a measure of functional disability and represents the current quality of life (92). Only 2 trials of the 20 trials examined in this study reported BI $(76,83)$. The results showed that PNS treatment significantly increased BI when compared to the CT $(\mathrm{MD}=-11.73 ; 95 \% \mathrm{CI}=-19.31,-4.16 ; P=0.002)$ (Figure 6). One trial involved 10 weeks of PNS treatment after $\mathrm{ICH}$ and the result showed no significant difference in BI at 14 days after PNS treatment $(P>0.05)$, but significant differences were observed at 28 days $(P<0.05)$ and 90 days after the treatment $(P<0.01)$,suggesting that a relatively long course of PNS treatment is necessary for significant improvement in functional recovery (76).

\section{$<72 \mathrm{H} \quad \mathrm{N} / \mathrm{A} \quad$ Mortality rate}

N/A Figure 7 shows the results of meta-analyses on the NDP. Six trials 3rd WS

WS

$\leq 48 \mathrm{H}$

\section{WS}

WS

$<48 \mathrm{H} \quad$ WS

WS

NR WS

WS

$\geq 7 D \quad$ HYTN

HYTN the six trials died $(6.7 \%)$. The mortality of ICH patients was significantly lower in the PNS group $(13 / 361$, or $3.6 \%)$ than in the CT group (35/354, or 9.9\%) (Peto OR $=2.78$; $95 \% \mathrm{CI}=1.52,5.08$; $P=0.0009)$. The trials were further divided into two subgroups depending on the time of PNS intervention: (1) three trials that started PNS treatment within 48 h of ICH onset $(74,84,88)$ and (2) four trials that started PNS treatment at or after 7 days of $\mathrm{ICH}$ onset $(72,78,87)$. One trial evaluated PNS intervention at both $48 \mathrm{~h}$ and 7 days after ICH onset (88). All trials assessed the mortality at the end of the treatment course. The duration of PNS treatment was 4 (72) and 6 (74) weeks, respectively, for 2 of the trials, and was 2 weeks for the other trials. The results showed significant reduction in the mortality of $\mathrm{ICH}$ patients treated with 
Table 2 | PNS treatment information of the 20 trials included in the meta-analyses.

\begin{tabular}{|c|c|c|c|c|}
\hline Authors & Interventions (sample size) Dosage & Durations & Observations & Adverse incidences (\%) \\
\hline Guo et al. (90) & $\begin{array}{l}\mathrm{PNS}(n=100): \mathrm{XST} \text { Inj } 200 \mathrm{mg} / \text { day } \\
\mathrm{CT}(n=100): \mathrm{MNT}, \mathrm{ST}\end{array}$ & 3 weeks & ER & NO \\
\hline He et al. (89) & $\begin{array}{l}\mathrm{PNS}(n=12): \mathrm{XSHT} \text { Inj } 140 \mathrm{mg} / \text { day } \\
\mathrm{CT}(n=12): \mathrm{MNT}, \mathrm{VC}, \mathrm{KCl}\end{array}$ & 2 weeks & IHV,IEV & NR \\
\hline Xu and Dong (72) & $\begin{array}{l}\mathrm{PNS}(n=42): \text { XST FDP } 400 \mathrm{mg} / \mathrm{day} \\
\mathrm{CT}(n=40): \mathrm{DH}, \mathrm{ICP}, \mathrm{ST}\end{array}$ & 4 weeks & ER,NDP & NR \\
\hline Li et al. (88) & $\begin{array}{l}\text { PNS }(n=55): \text { XST Inj } 600 \mathrm{mg} / \text { day } \\
\text { CT }(n=60): \text { AHT,CICP,ST }\end{array}$ & 2 weeks & ER,NDP,IHV & $\begin{array}{l}\mathrm{Sr}(5 \%) \\
\mathrm{NO}\end{array}$ \\
\hline Li and Yang (73) & $\begin{array}{l}\mathrm{PNS}(n=48): \text { XST FDPI } 400 \mathrm{mg} / \text { day) } \\
\mathrm{CT}(n=44): \text { CAS }\end{array}$ & 2 weeks & ER,NDS & NO \\
\hline Tian et al. (74) & $\begin{array}{l}\mathrm{PNS}(n=36): \mathrm{XST} \text { Inj } 200 \mathrm{mg} / \text { day } \\
\mathrm{CT}(n=30): \mathrm{MNT}, \mathrm{FRS}, \mathrm{ST}\end{array}$ & 6 weeks & ER,NDP,NDS,IHV & NR \\
\hline Xie et al. (76) & $\begin{array}{l}\mathrm{PNS}(n=24): \mathrm{XSHT} \text { Inj } 400 \mathrm{mg} / \text { day } \\
\mathrm{CT}(n=22): \mathrm{DH}, \mathrm{CICP}, \mathrm{ST}\end{array}$ & 10 weeks & $\mathrm{ER}, \mathrm{BI}$ & NO \\
\hline Chen et al. (77) & $\begin{array}{l}\mathrm{PNS}(n=22): \mathrm{XST} F \mathrm{FPI} 800 \mathrm{mg} / \text { day } \\
\mathrm{CT}(n=21): \mathrm{DH}, \mathrm{CICP}, \mathrm{AHT}, \mathrm{ST}\end{array}$ & 2 weeks & NDS & NO \\
\hline Dong and Wang (78) & PNS( $n=40):$ XST FDPI 400 mg/day & 2 weeks & ER,NDP & NR \\
\hline Zhang et al. (71) & $\begin{array}{l}\mathrm{CT}(n=38): \mathrm{DH}, \mathrm{CICP}, \mathrm{ST} \\
\mathrm{PNS}(n=65): \mathrm{LLT} \text { Inj } 250 \mathrm{mg} / \text { day } \\
\mathrm{CT}(n=65): \mathrm{MNT}, \mathrm{MGSO}_{4}, \mathrm{KCL} \\
\text { INS, Aceglutamide Inj }\end{array}$ & 2 weeks & ER & NO \\
\hline Song (82) & $\begin{array}{l}\text { PNS }(n=39): \text { XSHT Inj powder } 450 \text { mg/day } \\
\text { CT( } n=39): \text { CICP, AHT,ST }\end{array}$ & 4 weeks & IHV,IEV & NR \\
\hline Ding HY 2008 (83) & $\begin{array}{l}\mathrm{PNS}(n=15): \mathrm{XST} F D P I 200 \mathrm{mg} / \text { day } \\
\mathrm{CT}(n=17): \mathrm{AHT}, \mathrm{MNT}, \mathrm{CICP}, \mathrm{ST}\end{array}$ & 2 weeks & $\mathrm{BI}$ & NR \\
\hline Yuan HY 2008 (84) & $\begin{array}{l}\mathrm{PNS}(n=67): \mathrm{XST} \text { Inj } 750 \mathrm{mg} / \text { day } \\
\mathrm{CT}(n=66): \mathrm{MNT}, \mathrm{GFI}\end{array}$ & 2 weeks & ER,NDP & NO \\
\hline Ding and Geng (85) & $\begin{array}{l}\mathrm{PNS}(n=86): \text { XSHT Inj } 175 \mathrm{mg} / \text { day } \\
\mathrm{CT}(n=86): \mathrm{CICP}, \mathrm{AHT}, \mathrm{ST}\end{array}$ & 3 weeks & $\mathrm{ER}, \mathrm{IHV}, \mathrm{IEV}$ & NO \\
\hline Gao HY 2008 (86) & $\begin{array}{l}\mathrm{PNS}(n=28): \mathrm{XST} \text { Inj } 250 \mathrm{mg} / \text { day } \\
\mathrm{CT}(n=24): \mathrm{MNT}, \mathrm{CICP}, \mathrm{AHT}, \mathrm{ST}\end{array}$ & 2 weeks & NDS & NR \\
\hline Chen (87) & $\begin{array}{l}\mathrm{PNS}(n=61): \text { SQZG } 350 \mathrm{mg} / \mathrm{day} \\
\mathrm{CT}(n=60): \mathrm{CICP}, \mathrm{AHT}, \mathrm{ST}\end{array}$ & 2 weeks & $\mathrm{ER}, \mathrm{NDP}, \mathrm{HHV}$ & NR \\
\hline
\end{tabular}

$A H T$, anti-hypertensive treatment; BCA, brain cell activators; $B I$, Barthel index; $C A S$, citicoline sodium; CICP, control of intracerebral pressure; DH, dehydration; ER, effectiveness rate; DPI, freeze-dry powder injector; FRS, furosemide; GFI, glycerol and fructose injection; IEV, intracerebral edema volume; IHV, intracerebral hematoma

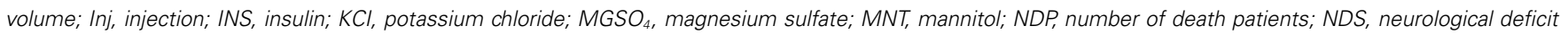

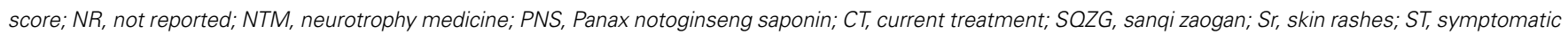
treatment; VC, vitamin C; XSHT, Xue Shuan Tong; XST, Xue Sai Tong (Xuesaitong). 
Table 3 | Detailed information of the hemorrhage sites of the 20 trials included in the meta-analyses.

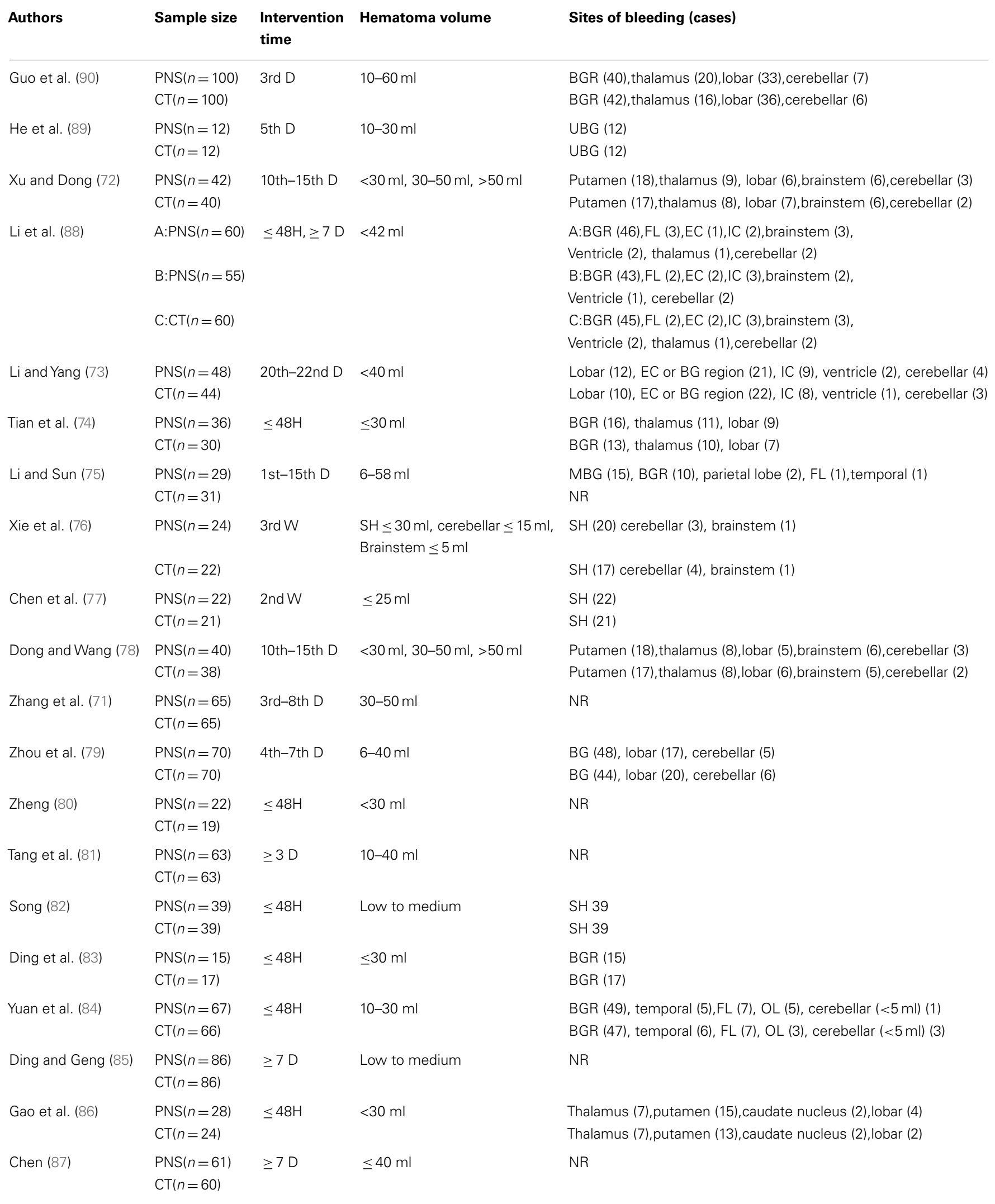

BG, basal ganglia; BGR, basal ganglia region; $C T$, current treatment; $E C$, external capsule; $F L$, frontal lobe; IC, internal capsule; MBG, medial basal ganglia; $O L$, occipital lobe; PNS, Panax notoginseng saponin; PL, parietal lobe; SH, supratentorial hemorrhage; UBG, unilateral basal ganglia. 


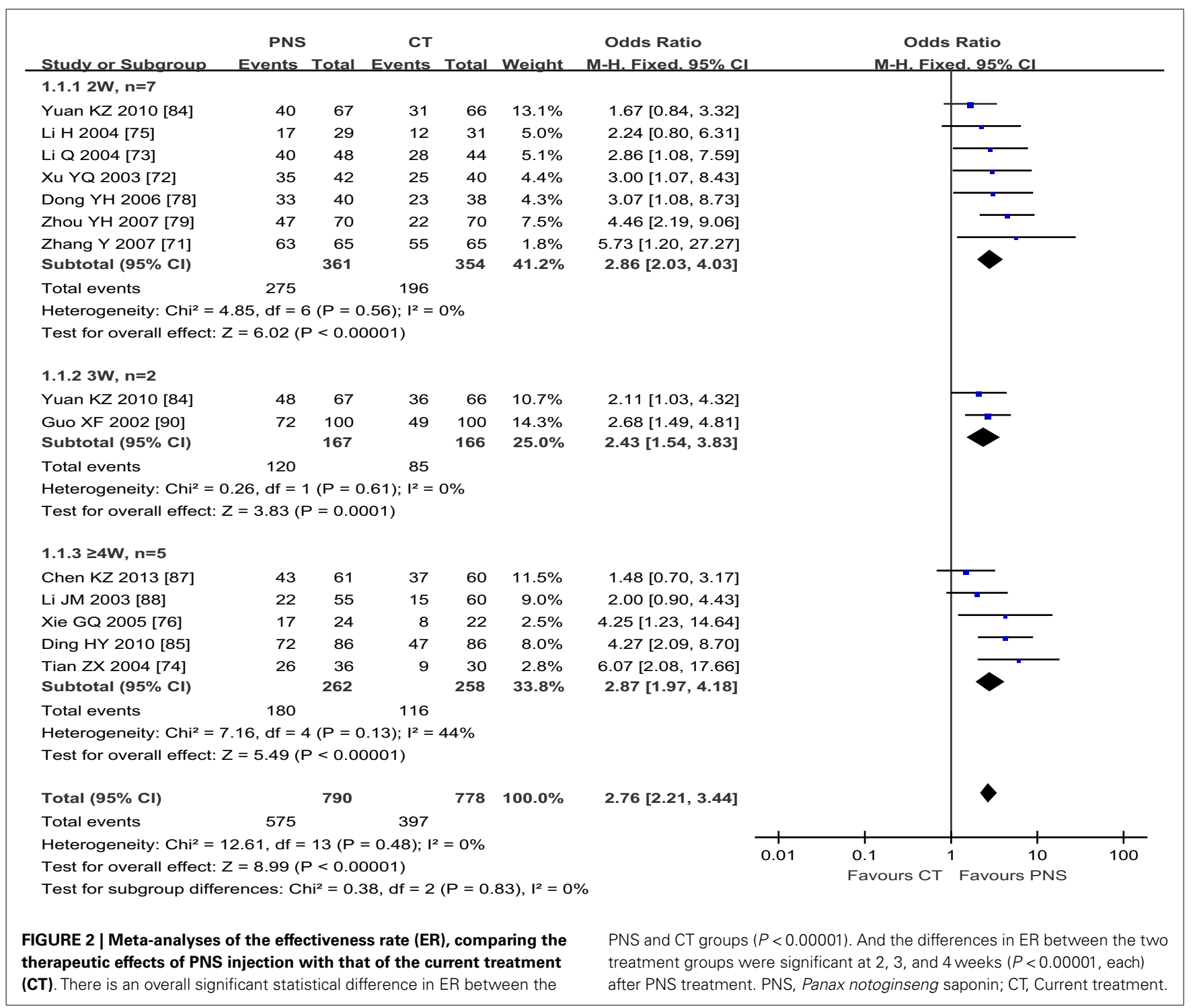

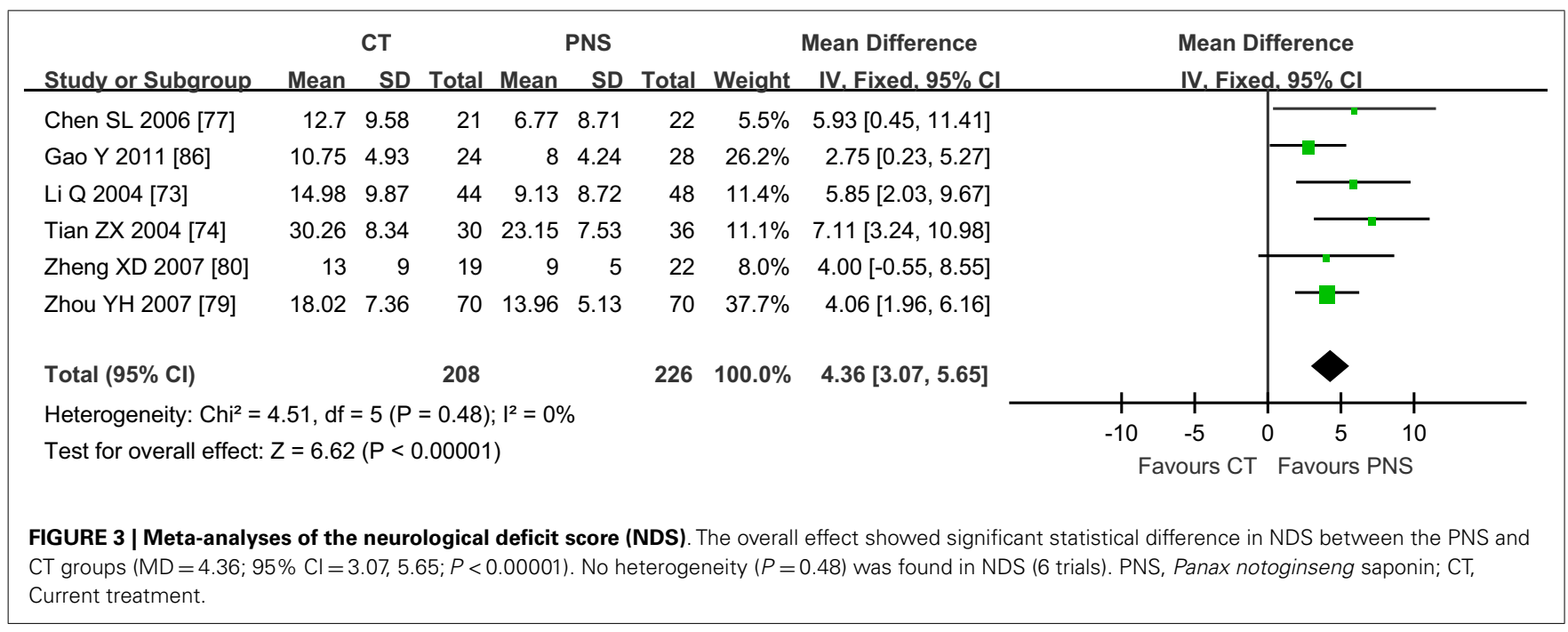




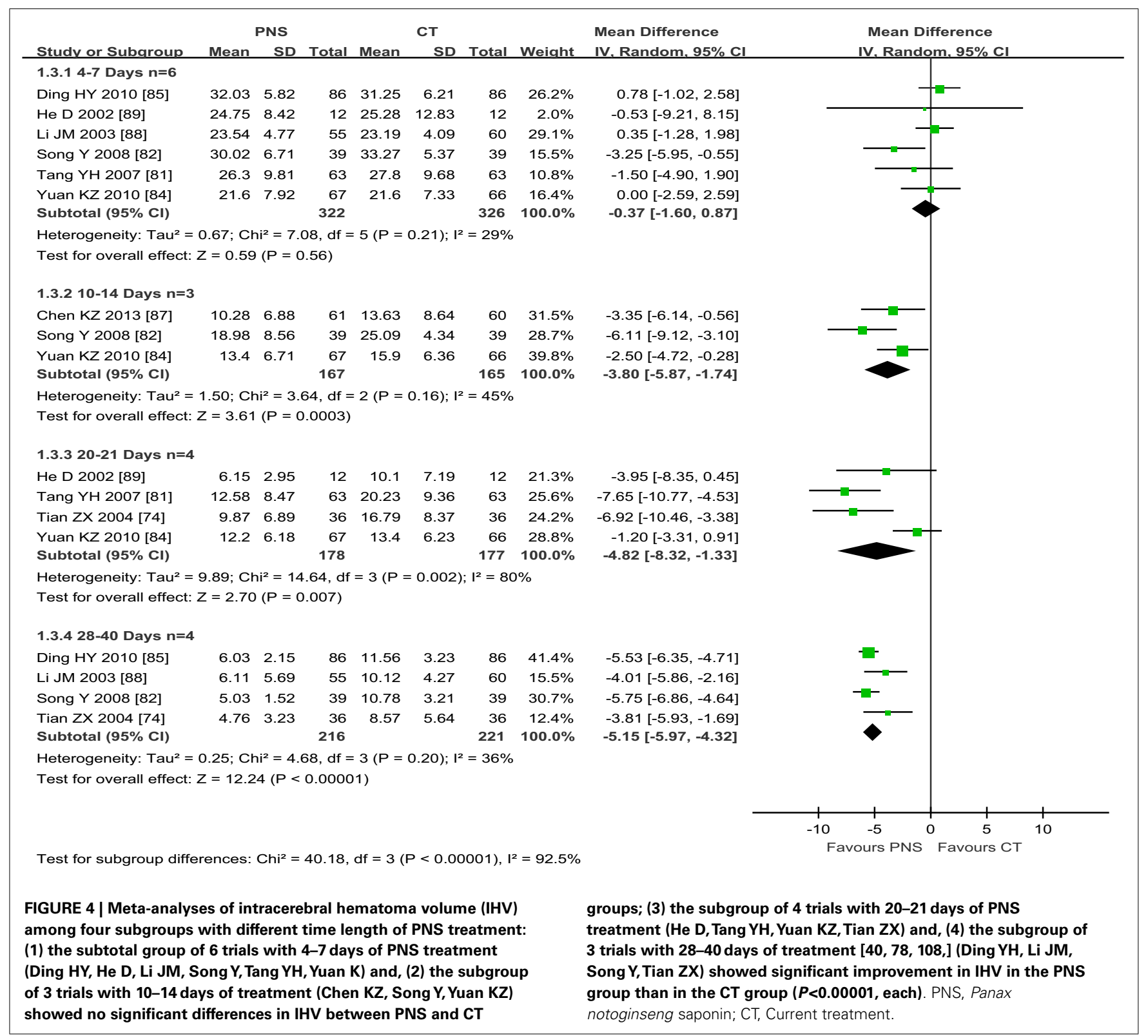

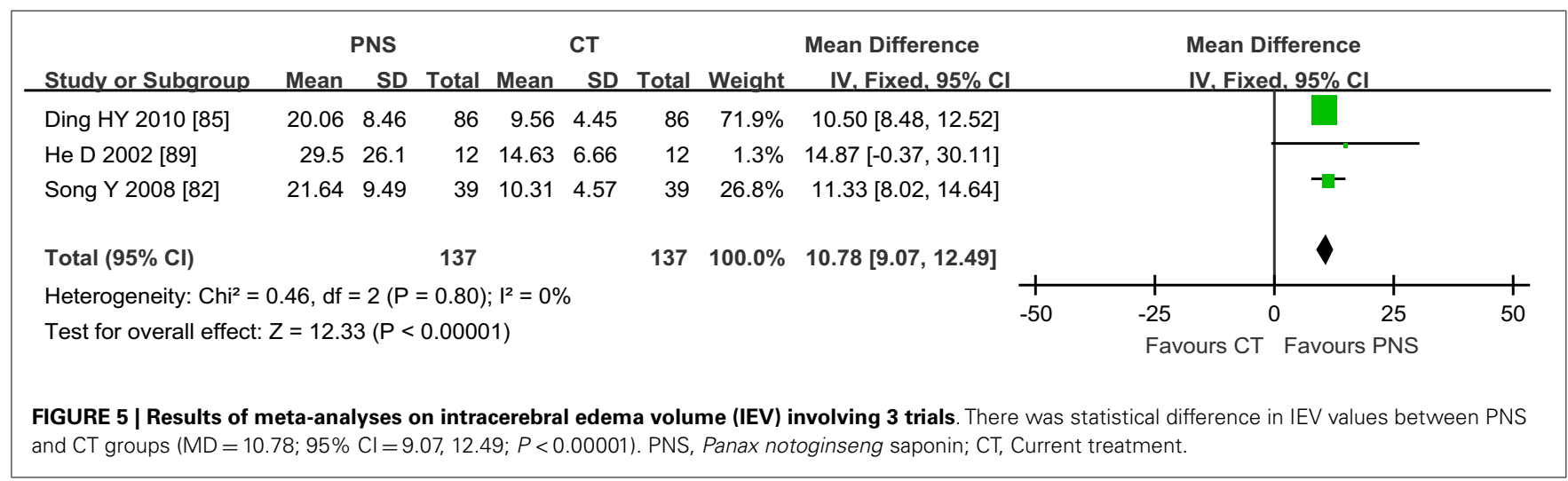




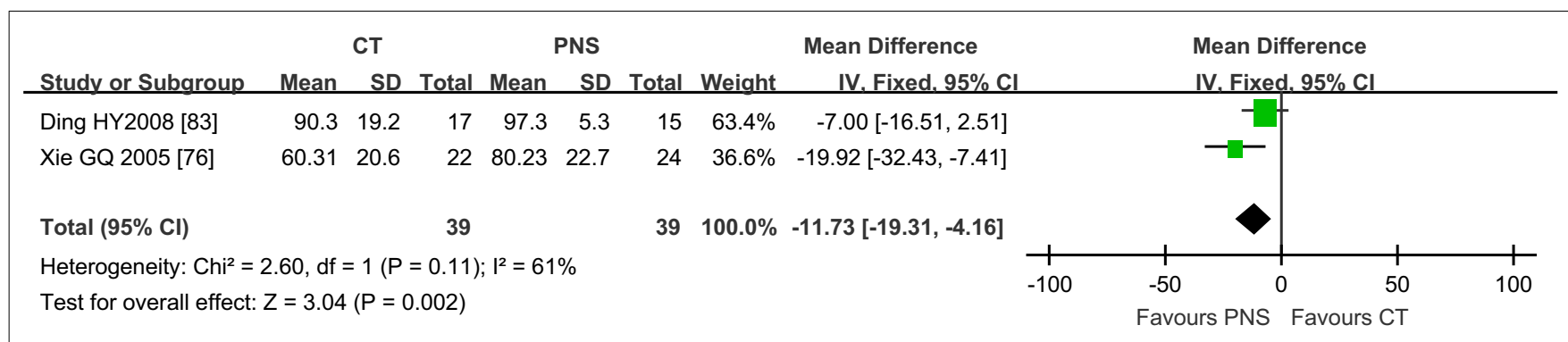

FIGURE 6 | Results of meta-analyses on Barthel index (BI) (2 trials). PNS treatment significantly increased BI compared to RT (MD =-11.73; $95 \%$ $\mathrm{Cl}=-19.31,-4.16 ; P=0.002$ ). PNS, Panax notoginseng saponin; CT, Current treatment.

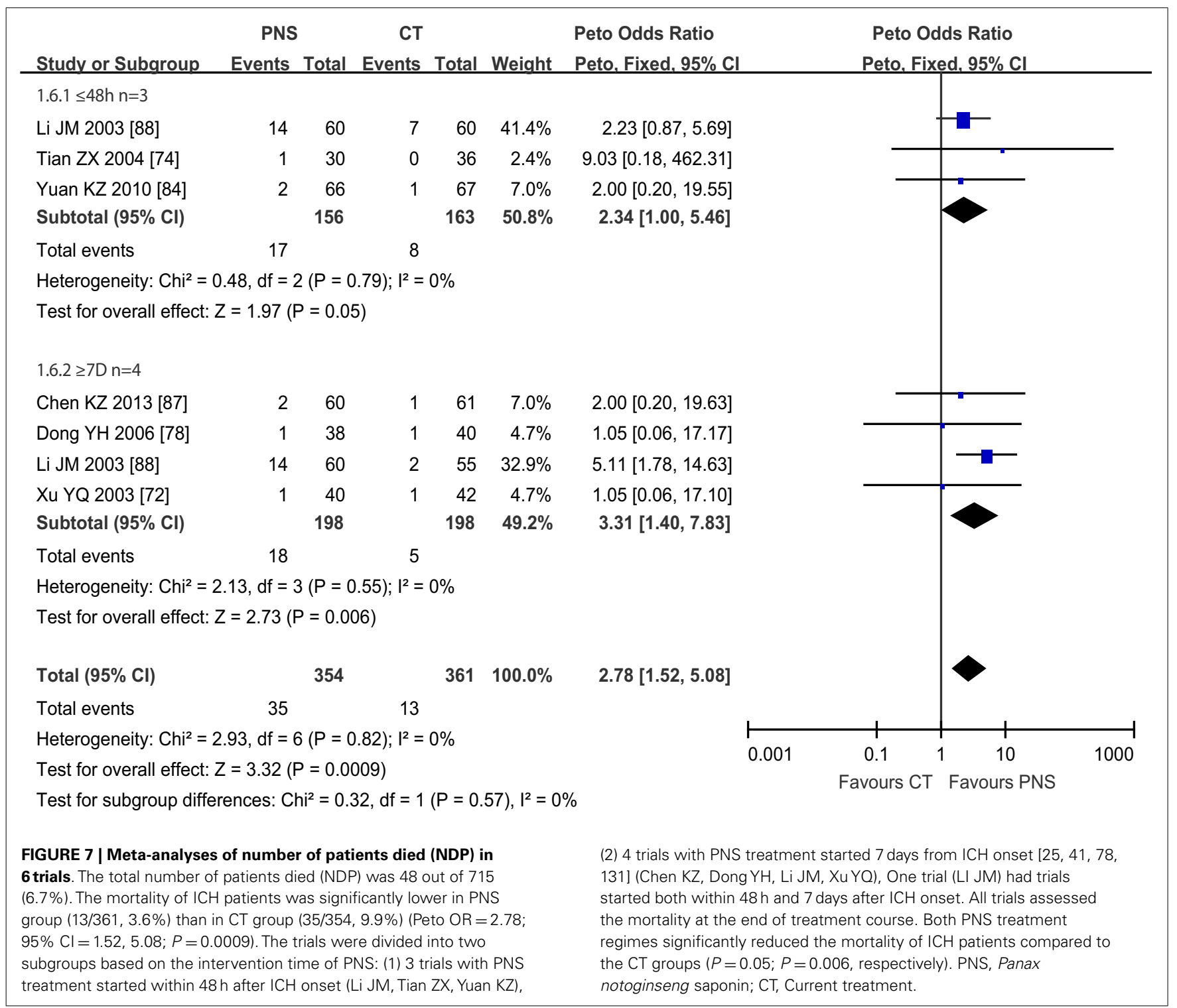

PNS within $48 \mathrm{~h}$ of $\mathrm{ICH}$ onset (Peto $\mathrm{OR}=3.31 ; 95 \% \mathrm{CI}=1.40$, 7.83; $P=0.006$ ), but no significant differences between the PNS and CT groups when PNS treatment started more than $48 \mathrm{~h}$ after
ICH onset $($ Peto $\mathrm{OR}=2.34 ; 95 \% \mathrm{CI}=1.00,5.46 ; P=0.05)$, suggesting that early PNS intervention is critical in reducing $\mathrm{ICH}$ mortality. 


\section{META-ANALYSIS ON SAFETY}

Seven trials reported incidences of adverse events $(73,76,77,84$, $85,88,90)$. Only one trial reported three cases of skin rashes related to PNS injection (56). No severe side effects were reported in the other six trials.

\section{QUALITY ASSESSMENT}

The quality assessment of the 20 included trials was evaluated in accordance with Jadad's scale. Four trials were assessed as highquality trials (scoring 3-5 marks) $(76,80,88,89)$, and the rest were assessed as low-quality trials (scoring 1-2 marks) owing to poor description on randomization and blindness in the papers. All trials mentioned randomization and dropout rate but only three of them described randomization methods $(76,80,88)$ and one trial mentioned single blinding in their methodological design (89).

\section{FUNNEL PLOTS}

To determine potential publication bias, funnel plot based on the effective rate was elaborated (Figure 8). A total of 13 trials reported the effect rate, which was categorized into three subgroups by the treatment outcome evaluating time: (1) 7 trials assessed the ER at the end of 2 weeks of treatment, (2) 2 trials assessed the ER at the end of 3 weeks, and (3) 5 trials assessed the ER at the end of 4 weeks.

\section{DISCUSSION}

Intracerebral hemorrhage is the leading cause of death and disability in people with TBI and stroke. So far, no proven drug is available for ICH. From the meta-analysis of multiple clinical trials involving PNS treatment for intracerebral hemorrhage (ICH), we found that ICH patients treated with PNS exhibited better outcomes than ICH patients that received current treatments in all aspects examined including the ER, the NDS, intracranial hematoma volume (IHV), intracranial edema volume (IEV), BI, and NDP. This finding is consistent with the knowledge that $P$. notoginseng is an effective medical herb for wound healing and bleeding.

\section{INTERVENTION TIME}

Because intracerebral bleeding is a major cause of mortality and morbidity in ICH patients, timely intervention is critical for saving lives and for better outcomes of the patients. P. notoginseng is a hemostatic drug that has been used to stop bleeding after gunshots and traumatic injuries since the Ming Dynasty (93). The non-protein amino acid dencichine and notoginsenoside Ft1 of PNS have been identified as the active hemostatic components of P. notoginseng (17).

The timing of PNS intervention varied among the 20 clinical trials examined. The earliest PNS intervention was given within $48 \mathrm{~h}$ of ICH onset $(74,80,82,84-86)$. These early treated patients had the following common characteristics: hemorrhaging of less than $30 \mathrm{ml}$, diagnosis of the precise bleeding site, no comas, and stable vital signs. The last PNS intervention began 3 weeks after ICH onset $(73,76)$. The majority of the patients, however, received PNS between 1 and 2 weeks after ICH onset. Only one trial examined the timing effect of PNS intervention on the outcome of ICH (88). In that trial, $60 \mathrm{ICH}$ patients received $600 \mathrm{mg} /$ day of PNS within $24 \mathrm{~h}$ after ICH onset, and $55 \mathrm{ICH}$ patients received $600 \mathrm{mg} /$ day of PNS injection 7 days after ICH onset. Both treatments lasted 14 days and both treatments improved the outcome

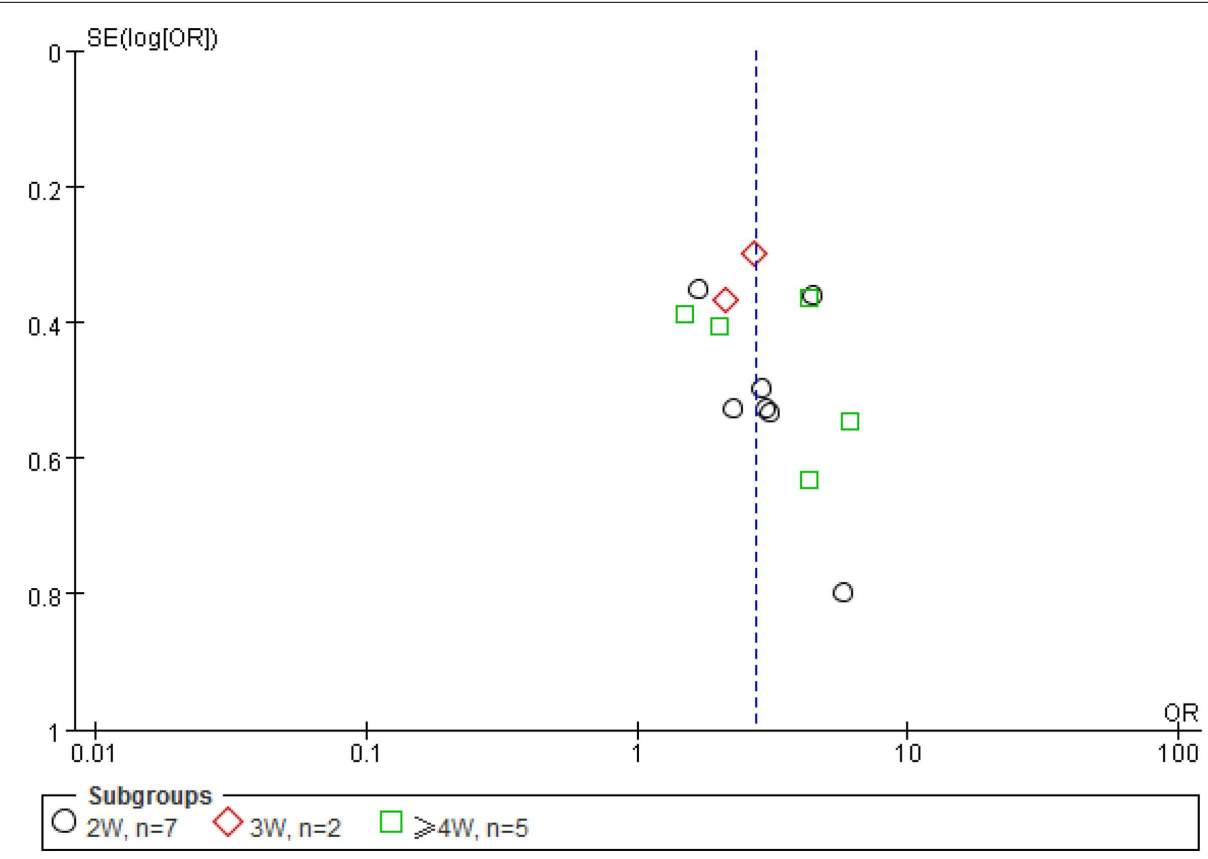

FIGURE 8 | A funnel plot was made using the data of effectiveness rate (ER). A total of 13 trials reported the effect rate, which was categorized into three subgroups by the evaluating time: (1) 7 trials assessed the ER at the end of 2 weeks of treatment, (2) 2 trials assessed the ER at the end of 3 weeks, and (3) 5 trials assessed the ER at the end of 4 weeks. Because of the small number of trials involved, the imperfect or asymmetrical funnel plot is not a reliable indication of potential publication bias in this case. 
of the ICH patients compared to the CT groups. No difference was found in the ER between the two PNS treatment groups $(P>0.5)$.

The results show that the death rate was two- to threefold lower in $\mathrm{ICH}$ patients that received PNS treatment within $48 \mathrm{~h}$ of $\mathrm{ICH}$ onset than those in the CT group ( $P=0.05$, Figure 6), suggesting early PNS intervention can be critical for saving the lives of ICH patients at risk of death.

Animal studies showed that PNS given at the third day of acute cerebral hemorrhage produced the best effects in reducing cerebral edema and hematoma volume in rats when compared to PNS administration given at other times (94). These authors reported up regulation of Bcl-2 expression in cerebral tissue after PNS treatment. Another animal model study showed that early PNS treatment after ICH onset can significantly suppress brain inflammation as reflected in reduced level of CAM- 1 and TNF- $\alpha$ expression in PNS-treated rats (95). Thus early PNS intervention could minimize ICH-induced brain inflammation and neuronal apoptosis, and facilitate the restoration of normal brain function.

\section{ADVERSE EVENTS, DOSAGE, AND ROUTE OF ADMINISTRATION}

Seven clinical trials included in this study reported the observation of adverse events $(73,76,77,84,85,88,90)$. Only three cases of skin rash were reported after PNS (XST) injection in one trial (56), accounting for $5.5 \%$ of the 55 PNS-treated patients of that trial (Table 1). This finding is in agreement with our previous study that the side effects of PNS use are relatively rare, with skin rash being the most common side effect of PNS use, accounting for more than $52 \%$ of all its adverse reactions (96).

There were considerable variations in the daily dose and total dose of PNS administration among the 20 clinical trials included in this study, with the lowest dose at $140 \mathrm{mg} /$ day, the highest dose at $800 \mathrm{mg} / \mathrm{day}$, and the average dose at $373.25 \pm 181.57 \mathrm{mg} /$ day. Most of the trials administered PNS at a dose range between 300 and $400 \mathrm{mg} /$ day, with 12 trials at a dose greater than $300 \mathrm{mg} /$ day. So far, there is no consensus about the ideal daily dose and total dose of PNS for ICH although 200-300 mg/day for 2-4 weeks are usually recommended by the manufacturers of medical PNS.

For the treatment duration and the total dose, some studies recommend a treatment course of 14 days (78), but others propose a 30-40-day treatment regime for achieving a better outcome $(74,97)$. Of the 20 clinical trials included in this study, 1 trial treated the ICH patients for 1 week, 2 trials for 11 weeks, 4 trials for 3 weeks, 3 trials for 4 weeks, and 1 trial for 6 weeks (Table 1). Our analysis shows that an increased duration of PNS treatment is linked to a better outcome of the intracerebral hemotoma volume (Figure 4). Significant improvement in IHV was found only after 10 or more days of PNS treatment. It appears that a minimum of 2-4 weeks of treatment is required to achieve significant improvement in the outcome of patients with acute ICH. A longer treatment could bring additional benefit but with an additional medical cost.

\section{MORTALITY}

Significantly reduced morbidity and mortality are the key indexes of successful treatment of intracerebral hemorrhage. Of the 20 clinical trials, 3 trials reported short-term mortality in $\mathrm{ICH}$ patients who received PNS treatment within $48 \mathrm{~h}$ after ICH onset
$(74,84,88)$ (Figure 7), and 4 trials reported short-term mortality in $\mathrm{ICH}$ patients who received PNS treatment 7 days after $\mathrm{ICH}$ onset (Figure 7). Both treatment regimens exhibited a more significantly reduced the death rate in the PNS group than in the CT group (by $>2, P<0.05$, and $>3$-fold, $P<0.01$, respectively). This is consistent with a recent report that certain herbal ingredients of traditional Chinese medicine (TCM) could stimulate the activation of blood, resolve hemostasis, and reduce acute $\mathrm{ICH}$-induced short-term mortality (10).

\section{QUALITY OF LIFE}

The quality of life of the ICH patients is closely associated with the severity of the disease and the efficacy of the treatment $(98,99)$ and is often assessed using the NDS and the BI. Of the 20 trials included in the present study (with a total of 1,891 ICH patients), only 2 trials measured BI (Figure 4) and reported improved activities of daily living in the PNS-treated ICH patients compared to the patients in the CT group.

Neurological deficit score is an important index for the diagnosis of symptom severity and functional recovery of the patients. Three of the trials included in this study reported NDS at 7, 15, 21, 28, and 30 days after PNS treatment $(74,77,86)$ (Figure 2). The results showed that it took at least 21 days for the PNS treatment to produce noticeable improvements in $\mathrm{ICH}$ patients when compared with the CT groups $(74,77,86)$. That improvement in terms of NDS, however, became significant at 28 days in one study (86) and 40 days in another (74) after the start of PNS treatment $(P<0.05, P<0.01$, respectively).

\section{FUNNEL PLOTS}

The funnel plot is often used to check for the existence of publication bias in meta-analyses, assuming that the largest studies will be plotted near the average, and smaller studies will be spread evenly on both sides of the average, creating a roughly funnel-shaped distribution. Deviation from this shape can indicate publication bias. Although the funnel plot appeared asymmetrical in this study, it may reflect the fact of unbalanced and very limited data of each group ( $2 \mathrm{~W} n=7,3 \mathrm{~W}, n=2,4 \mathrm{~W}, n=5)$ (Figure 8) used for the plot rather than a true publication bias as eight samples from each group is considered minimal (while more than 20 is preferred) number required for a meaningful funnel plot, and a less funnel plot may give a wrong impression of publication bias if high precision studies are different from low precision studies with respect to effect size (e.g., due to different populations examined) (100). The appearance of the funnel plot can also alter substantially depending on the scale on the $y$-axis (101).

\section{MECHANISM OF PNS ACTION}

Intracerebral hemorrhage can disrupt cerebral blood flow, energy metabolism, and the integrity of the blood-brain barrier (BBB), resulting in edema, inflammation, apoptosis, neurological dysfunction, and often death. Acute and chronic macro- and micro-bleeding and thrombosis are the primary determinants of hematoma and edema development. Agents that can effectively control the bleeding and reduce hematoma and edema hold the promise to become effective therapies for $\mathrm{ICH}$ and have been the major treatment targets of multiple international ongoing randomized control trials of ICH (102). 
Our meta-analysis shows that total $P$. notoginseng saponin extract (PNS) could be a therapeutic agent for ICH because it can significantly attenuate edema and hematoma in ICH patients. This is in line with recent reports that PNS (XST) injection treatment ( $175 \mathrm{mg} /$ day) for 2 weeks significantly improved hematoma absorption and neurological function in 32 acute ICH patients compared to 29 RT controls (103). PNS has also been shown to improve microcirculation around the hematoma ischemic area, promote the absorption of hematoma, slow down and inhibit brain edema development, and significantly shorten the time for the edema to disappear (104).

The mechanism of PNS' neuroprotection against ICH injury remains to be fully understood. It may involve differential protective activities of various ginsenosides and notoginsenosides on hemostasis, anti-coagulant, anti-thrombotic, platelet aggregation and complement activation, hemorheology, blood viscosity and hematocrit, vasodilation, microcirculation, energy metabolism, oxidative stress, inflammation, and immune function. Some of the recently published PNS actions are presented below as they may be relevant or potential mechanisms of PNS in ICH.

\section{HEMOSTATIC EFFECTS OF PNS}

Spontaneous acute and chronic macro- and micro-bleeding contribute directly to hematoma growth in TBI and ICH patients and are linked with symptom severity, recurrence, and poor outcome $(105,106)$. Preclinical TBI studies showed that the extent of ICH acquired during acute and subacute phases (3 h, 3, 9, and 23 days) post-ICH can predict the functional and histopathological outcome in rats $6-12$ months later and is correlated with the final cortical atrophy $(P<0.05)$, hippocampal atrophy $(P<0.01)$, and memory deficits $(P<0.01)(107)$.

At least two PNS components, i.e., dencichine and notoginsenoside Ft1 have been identified to possess hemostatic properties that could block or minimize bleeding and hematoma expansion after ICH onset $(17,108)$. Dencichine is a bioactive non-protein therapeutic amino acid found in $P$. notoginseng. At low concentrations, dencichine has hemostatic and platelet-enhancing activity, but at high concentrations, it is neurotoxic (108). Decichine enhances hemostasis of activated platelets via AMPA receptors (109). Notoginsenoside Ft1 is a potent procoagulant that can induce dose-dependent and ADP-induced platelet aggregation, increase plasma coagulation indexes, decrease tail-bleeding time, and increase thrombogenesis and cytosolic $\mathrm{Ca}(2+)$ accumulation. Dencichine and notoginsenoside Ftl may underlie the hemostatic mechanism of PNS during the acute and subacute phases of ICH.

\section{ANTI-THROMBOSIS, FIBRINOLYSIS, AND ANTI-COAGULATION MECHANISM: ROLE OF NITRIC OXIDE}

Patients with TBI and resultant intracranial hemorrhage (ICH) are at high risk for developing venous thromboembolism (VTE) (110). Intrahematomal blood clotting is also a pathogenetic factor in hyperacute perihematomal edema formation (111). There is an increasing use of anti-platelets and/or anti-coagulants in the treatment of blood clotting and hyperviscosity in $\mathrm{ICH}$ and there is some evidence of therapeutic effects in animal models of $\mathrm{ICH}$
(112-115). Several compounds of PNS including adenosine and guanosine, ginsenoside Rh1, F1, Rg1, and Rg2 have anti-platelet and anti-coagulant activities, with adenosine and guanosine and the ginsenosides as the main anti-platelet aggregation compounds of PNS (116-118).

One study showed that sanchinoside Rg1 markedly inhibited experimental thrombosis formation by enhancing the function of fibrinolysis system and stimulating vascular endothelial cells to release nitric oxide (NO) (119). Ginsenoside Rb1 can also reverse oxidative stress- and ischemia-related umbilical endothelial dysfunction and myocardial injury through upregulation of the endothelia NO synthase (eNOS) pathway in diabetes rat model $(120,121)$.

\section{PNS HAS ANTI-HYPERTENSION ACTIVITY}

Hypertenison is a critical pathological factor in triggering ICH onset. Notoginsenoside Ft1 activates both glucocorticoid and estrogen receptors to induce endothelium-dependent, NOmediated relaxations in rat mesenteric arteries (122).

\section{PNS INHIBITS COMPLEMENT ACTIVATION}

PNS could improve the outcome of acute ICH by suppressing the complement 3 (C3)-mediated pathway. Activation of complement cascades plays an important role in anaphylatoxin-mediated inflammation, secondary toxicity, and brain damage after ICH $(24,123)$. Studies have shown that PNS (co-)therapy inhibited the enhancement of blood complement $\mathrm{C} 3$ levels in experimental ICH (86). Significant reduction in circulation complement (C3) was found in 43 rheumatoid arthritis patients treated with PNS for 28 days, and which was associated with improved clinical symptoms such as joint swelling index when compared to the control subjects (50). This could be a potential mechanism underlying the decreased volume of intracerebral edema in the patients receiving PNS treatment group reported in the three clinical trials (Figure 5). In ICH-induced local tissue inflammation, C3 promotes the adhesion, exudation and translocation of inflammatory cells, and stimulates the secretion of large amounts of inflammatory mediators such as TNF- $\alpha$ and IL- $1 \beta$, resulting in an increased inflammatory response and brain damage. These responses are absent in mice deficient in C3 activity and show reduced inflammatory cell infiltration, brain edema formation, and improved neurologic outcome after experimental ICH $(124,125)$.

\section{PNS PROTECTS BBB INTEGRATION}

Blood-brain barrier disruption is a hallmark of ICH-induced brain injury and contributes to edema formation, the influx of leukocytes, and the entry of potentially neuroactive agents into the perihematomal brain, all of which can contribute to brain injury. Factors implicated in BBB disruption include: inflammatory mediators (e.g., cytokines and chemokines), thrombin, hemoglobin breakdown products, oxidative stress, complement proteins, and matrix metalloproteinases, etc. $(126,127)$. Two studies have shown that ginsenoside $\mathrm{Rg} 1$ provides neuroprotection against BBB disruption, edema formation, and neurological injury in rat models of cerebral ischemia/reperfusion through the downregulation of aquaporin 4 expression and anti-apoptosis pathways $(38,128)$. 


\section{PNS PROTECTS AGAINST ISCHEMIA/REPERFUSION, AND STIMULATES ANGIOGENESIS}

Neurons are oxygen sensitive and are vulnerable to ischemicreperfusion injury after ICH. Experimental studies have shown that PNS and ginsenosides Rb1 and Rb3 can provide significant protection against ischemia/reperfusion injury in rodent brains $(36,129)$, cardiomyocytes (130, 131), and kidneys (132). Ginsenoside Rb1 prevents homocysteine-induced endothelial dysfunction via PI3K/Akt activation and PKC inhibition (133). Ginsenoside Rg1 enhances angiogenesis after hypoxia ischemia brain damage in neonatal rats and in diabetic mice, in part through hypoxia-inducible factor (HIF-1a), glucocorticoid receptor (GR), and fibroblast growth factor receptor (VEGFR)-mediated pathways (134-138), and enhances the resistance of hematopoietic stem/progenitor cells to radiation-induced aging in mice (38). Notoginsenoside Ftl promotes angiogenesis via HIF-1 $\alpha$ mediated VEGF secretion and the regulation of PI3K/AKT and Raf/MEK/ERK signaling pathways (139). PNS also enhances VEGF signals and promotes angiogenesis derived from rat bone marrow and mesenchymal stem cells (140) as well as inhibit ischemia-induced apoptosis by activating the PI3K/Akt pathway in cardiomyocytes (141).

\section{PNS STIMULATES STEM CELL PROLIFERATION AND DIFFERENTIATION}

Cognitive impairment is common and is linked to neuronal cell loss after ICH (92). PNS could promote functional recovery of ICH patients through stimulating stem cell proliferation and differentiation. Studies have shown that ginsenoside Rb1 can improve spatial learning and memory by stimulating neurogenesis in the hippocampal subregions of rats (22) and that ginsenoside Rd can stimulate the proliferation of rat neural stem cells in vivo and in vitro (28). Ginsenoside Rgl stimulates the proliferation and differentiation of human dental pulp stem cells and facilitates neural differentiation of mouse embryonic stem cells via the GR-dependent signaling pathway $(31,32)$, which promotes peripheral nerve regeneration in the rat model of nerve crush injury (142) and improves spatial learning-memory in dementia rats after bone marrow mesenchymal stem cell transplant (21). Ginsenoside Rg1 mediates microenvironmentdependent endothelial differentiation of human mesenchymal stem cells (143).

\section{PNS PROTECTS MICROCIRCULATION FROM ISCHEMIA/REPERFUSION-INDUCED INJURY}

PNS treatment improved microcirculation around the hematoma ischemic area, promoted the absorption of hematoma, slowed down and inhibited brain edema development, and significantly shortened the time for the edema to disappear (104). Notoginsenoside R1 can attenuate ischemia/reperfusion (I/R)induced microvascular hyperpermeability, inflammatory cytokine production, NF-kB activation, leukocyte rolling and adhesion, the expression of E-selectin in endothelium and CD18 in neutrophils, loss of tight junction proteins, and deficit in energy metabolism during I/R in rats $(144,145)$. Ginsenosides Rb1 and $\mathrm{Rg} 1$, and notoginsenoside $\mathrm{R} 1$ have been shown to protect lipopolysaccharide-induced microcirculatory disturbance in rat mesentery (146).

\section{PNS HAS ROS-SCAVENGER, ANTIOXIDATION, AND ANTI-APOPTOSIS PROPERTIES}

PNS has been shown to be a potent antioxidant in various experimental models. PNS induces thioredoxin-1 expression and prevents 1-methyl-4-phenylpyridinium ion-induced neurotoxicity (147). Ginsenoside Rb1 directly scavenges hydroxyl radicals and hypochlorous acid (103) and inhibits apoptosis in hydrogen peroxide-treated chondrocytes by stabilizing mitochondria and inhibiting Caspase-3 (148). Ginsenoside Rb1 also prevented $\mathrm{MPP}(+)$-induced apoptosis in PC12 cells by activating estrogen receptors and ERK1/2/Akt pathways, and inhibiting SAPK/JNK/p38 MAPK pathways (149). Ginsenoside Rb1 protects against oxidative damage and renal interstitial fibrosis in rats with unilateral ureteral obstruction (150), and against beta-amyloid protein(1-42)-induced neurotoxicity in cortical neurons and in PC12 cells $(30,33)$ as well as against hypoxia and oxidative stress in rat retinal ganglion cells (29).

Ginsenoside Rd appears to be a superior neuroprotector with a wide therapeutic window in experimental stroke (151). Ginsenoside $\mathrm{Rd}$ attenuates redox imbalance, improves stroke outcome following focal cerebral ischemia in aged mice (152), and attenuates early oxidative damage and sequential inflammatory responses after transient focal ischemia in rats (153). Ginsenoside Rd prevents glutamate-induced apoptosis in rat cortical neurons (154), and promotes glutamate clearance by up-regulating the expression of glial glutamate transporter proteins (155). Ginsenoside-Rd exhibits anti-inflammatory activities through the enhancement of antioxidant enzyme activities and the inhibition of JNK and ERK activation in vivo (156).

Ginsenoside Rg1 protects against hydrogen peroxide-induced cell death in PC12 cells via the inhibition of NF-kB activation (157), reduction of nigral iron levels in MPTP-treated C57BL6 mice by regulation of iron transport proteins (158), and protection against beta-amyloid peptide-induced human endothelial cellapoptosis by activation of the GR-ERK signaling pathway (159). Oral Rg1 supplementation strengthens the antioxidant defense system against exercise-induced oxidative stress (160) and protects the liver against exhaustive exercise-induced oxidative stress in rats (161).

\section{PNS HAS ANTI-INFLAMMATION PROPERTIES}

Ginsenoside Rbl shows anti-neuroinflammation effects in rat models of Alzheimer's disease (20) and prevents interleukin-1binduced inflammation and apoptosis in human articular chondrocytes (162). Ginsenoside Rd inhibits the expression of iNOS and COX-2 by suppressing NF-kB in LPS-stimulated RAW264.7 cells and in mouse livers (163), and attenuates neuroinflammation in cultured dopaminergic neurons (164). Ginsenoside Re ameliorates inflammation by inhibiting the binding of lipopolysaccharides to TLR4 on macrophages (165). Ginsenoside Rg1 improves survival in a murine model of polymicrobial sepsis by suppressing the inflammatory response and apoptosis of lymphocytes (166). Ginsenoside Rg1 improves streptozocin (STZ)-induced diabetic nephropathy in rats by suppressing inflammatory reactions and expression of ectodermal dysplasia and TGF-beta (167). PNS also suppresses inflammation in a collagen-induced arthritis model (146). 


\section{ANTI-HYPERGLYCEMIA AND ANTI-HYPERLIPIDEMIC EFFECTS OF PNS}

Hyperglycemia is associated with poor outcome in patients with TBI and ICH and in experimental models of ICH $(93,168,169)$. PNS has hypolipidemic and antioxidant activities in rats with high-fat diets (170). Ginsenoside Rb1 has antiobesity and antihyperglycemic effects in rats (171). Ginsenoside Rb2 exerts its antidiabetic effects via activation of AMPK (172). Ginsenoside Rb2 lowers cholesterol and triacylglycerol levels in 3T3-L1 adipocytes under high cholesterol or fatty acids culture conditions (173). Ginsenoside Re reverses insulin resistance in muscles of high-fat diet rats (174). Ginsenoside Rg1 promotes glucose uptake through the activated AMPK pathway in insulin-resistant muscle cells (175).

\section{MANY COMPONENTS AND MANY MECHANISMS}

Cerebral hemorrhage causes brain damage through multiple mechanisms, with spontaneous bleeding, hematoma development and perihematoma edema formation as the main factors contributing to the poor outcome of ICH. Effective therapies for ICH should be able to target all these factors. Our meta-analysis and literature review suggest that PNS is an effective therapy for acute $\mathrm{ICH}$, and potentially functions through multiple mechanisms. Its most notable effects include hemostatic and anti-thrombotic effects, hemodynamic and hemorheological effects, angiogenesis and stem cell promoting effects, anti-hyperglycemia and antihyperlipidemia effects, and antioxidant and anti-inflammation effects, etc. Additionally, the strong tonic effects of PNS (176) could be beneficial to ICH patients, who are often weak and fragile during the recovery phase. A double-blind, double-dummy, randomized, and parallel-controlled study showed that 8 weeks after the onset of cerebral infarction, treatment of PNS tablets for 4 weeks significantly improved the outcome of the patients compared to the control treatments (177).

There are limitations to this research. Not all of the clinical trials analyzed were of high quality nor did they all include each desirable outcome evaluation. The number of ICH patients in each trial was often less than 100 and no long-term outcome data of the ICH patients were available. These and other factors should be controlled in further large scale studies so that the therapeutic effect of PNS can be better evaluated.

In conclusion, meta-analysis of the clinical trials suggests that PNS is superior to current treatment for acute ICH with minimal side effects. PNS could be an alternative therapy for acute ICH patients with a hemorrhagic volume of less than $30 \mathrm{ml}$. More clinical trials with better experimental designs could be conducted in the US and Europe to verify and extend the current findings and to determine the long-term effects of $P$. notoginseng on the recovery and recurrence of $\mathrm{ICH}$ patients.

\section{ACKNOWLEDGMENTS}

Ms. Jillian W. Wen edited the manuscript. Mr. Zhongjian Chen of Panax Notoginseng Research Institute of Wenshan State/Region, Yunnan Province, China provided the artistic drawing of notoginseng. This study is supported, in part, by a grant from the Department of Education, Guangxi Zhuang Autonomous Region, P.R. China (New Century Higher Education Reform program in Guangxi: Research and Practice of English Curriculum System in Nursing at Guangxi Traditional Chinese Medicine College) (2011JGZD. 014) (to Dongying Xu).

\section{SUPPLEMENTARY MATERIAL}

The Supplementary Material for this article can be found online at http://www.frontiersin.org/Journal/10.3389/fneur.2014.00274/ abstract

\section{REFERENCES}

1. Ghajar J. Traumatic brain injury. Lancet (2000) 356:923-9. doi:10.1016/S01406736(00)02689-1

2. Langlois JA, Rutland-Brown W, Thomas KE. Traumatic Brain Injury in the United States: Emergency Department Visits, Hospitalizations, and Deaths. Atlanta (GA): Centers for Disease Control and Prevention, National Center for Injury Prevention and Control (2006).

3. Hyder AA, Wunderlich CA, Puvanachandra P, Gururaj G, Kobusingye OC. The impact of traumatic brain injuries: a global perspective. NeuroRehabilitation (2007) 22(5):341-53.

4. Hanlon RE, Demery JA, Kuczen C, Kelly JP. Effect of traumatic subarachnoid haemorrhage on neuropsychological profiles and vocational outcome following moderate or severe traumatic brain injury. Brain Inj (2005) 19:257-62. doi:10.1080/02699050400004955

5. Harvey LA, Close JC. Traumatic brain injury in older adults: characteristics, causes and consequences. Injury (2012) 43:1821-6. doi:10.1016/j.injury.2012. 07.188

6. Edwards P, Arango M, Balica L, Cottingham R, El-Sayed H, Farrell B, et al. Final results of MRC CRASH, a randomised placebo-controlled trial of intravenous corticosteroid in adults with head injury-outcomes at 6 months. Lancet (2005) 365:1957-9. doi:10.1016/S0140-6736(05)66552-X

7. Vogel T, Ockert B, Krötz M, Linsenmaier U, Kirchhoff C, Pfeifer KJ, et al. [Progredient intracranial bleeding after traumatic brain injury. When is a contro CCT necessary?]. Unfallchirurg (2008) 111:898-904. doi:10.1007/s00113-0081502-0

8. Maas AI, Marmarou A, Murray GD, Teasdale SG, Steyerberg EW. Prognosis and clinical trial design in traumatic brain injury: the IMPACT study. J Neurotrauma (2007) 24:232-8. doi:10.1089/neu.2006.0024

9. MRC CRASH Trial Collaborators, Perel P, Arango M, Clayton T, Edwards P, Komolafe E, et al. Predicting outcome after traumatic brain injury: practical prognostic models based on large cohort of international patients. BMJ (2008) 336:425-9. doi:10.1136/bmj.39461.643438.25

10. Chen J, Zhang XY. Systematic evaluation of activating blood to resolve stasis for acute intracerebral hemorrhage mortality. Tradit Chin Emerg Med (2011) 20:1273-5.

11. Murray CJ, Lopez AD. Global mortality, disability, and the contribution of risk factors: global burden of disease study. Lancet (1997) 349:1436-42. doi:10.1016/S0140-6736(96)07495-8

12. van Asch CJ, Luitse MJ, Rinkel GJ, van der Tweel I, Algra A, Klijn CJ. Incidence, case fatality, and functional outcome of intracerebral haemorrhage over time, according to age, sex, and ethnic origin: a systematic review and meta-analysis. Lancet Neurol (2010) 9:167-76. doi:10.1016/S1474-4422(09) 70340-0

13. Feigin VL, Lawes CM, Bennett DA, Barker-Collo SL, Parag V. Worldwide stroke incidence and early case fatality reported in 56 population-based studies: a systematic review. Lancet Neurol (2009) 8:355-69. doi:10.1016/S1474-4422(09) 70025-0

14. Balami JS, Buchan AM. Complications of intracerebral haemorrhage. Lancet Neurol (2012) 11:101-18. doi:10.1016/S1474-4422(11)70264-2

15. Skolarus LE, Morgenstern LB, Zahuranec DB, Burke JF, Langa KM, Iwashyna TJ. Acute care and long-term mortality among elderly patients with intracerebral hemorrhage who undergo chronic life-sustaining procedures. J Stroke Cerebrovasc Dis (2013) 22:15-21. doi:10.1016/j.jstrokecerebrovasdis. 2011.05.025

16. Rodríguez-Yáñez M, Castellanos M, Freijo MM, López Fernández JC, MartíFàbregas J, Nombela F, et al. Clinical practice guidelines in intracerebral haemorrhage. Neurologia (2013) 28:236-49. doi:10.1016/j.nrl.2011.03.010

17. Gao B, Huang L, Liu H, Wu H, Zhang E, Yang L, et al. Platelet P2Y12 receptors are involved in the haemostatic effect of notoginsenoside Ft1, a saponin isolated from Panax notoginseng. Br J Pharmacol (2014) 171:214-23. doi:10.1111/bph. 12435

18. Liang XH, Yong J, Man R. Notoginsenoside Rgl on experimental thrombosis, platelet aggregation and platelet free calcium levels affect. Chin J Pharmacol Toxicol (1998) 12:40-2. 
19. Liu HZ, Pang J, Wang ZL, Ying GH, Li SR. [Studies of the effects of Gynura segetum and Panax notoginseng on the ultrastructure of platelets in guinea-pigs]. Yao Xue Xue Bao (1982) 17:801-8.

20. Wang Y, Liu J, Zhang Z, Bi P, Qi Z, Zhang C. Anti-neuroinflammation effect of ginsenoside Rbl in a rat model of Alzheimer disease. Neurosci Lett (2011) 487:70-2. doi:10.1016/j.neulet.2010.09.076

21. Wu W, Yang JQ, He ZY. [Effect of ginsenoside Rg1 on the spatial learningmemory ability in dementia rats after transplanted with bone marrow mesenchymal stem cells]. Zhongguo Zhong Xi Yi Jie He Za Zhi (2011) 31:799-802.

22. Liu L, Hoang-Gia T, Wu H, Lee MR, Gu L, Wang C, et al. Ginsenoside Rb1 improves spatial learning and memory by regulation of cell genesis in the hippocampal subregions of rats. Brain Res (2011) 1382:147-54. doi:10.1016/j.brainres.2011.01.051

23. An DS, Wang L, Kim MS, Bae HM, Lee ST, Im WT. Solirubrobacter ginsenosidimutans sp. nov., isolated from soil of a ginseng field. Int J Syst Evol Microbiol (2011) 61:2606-9. doi:10.1099/ijs.0.028431-0

24. Bai Y, Hu Y, Wu Y, Zhu Y, He Q, Jiang C, et al. A prospective, randomized, single-blinded trial on the effect of early rehabilitation on daily activities and motor function of patients with hemorrhagic stroke. J Clin Neurosci (2012) 19:1376-9. doi:10.1016/j.jocn.2011.10.021

25. Liu YW, Zhu X, Li W, Lu Q, Wang JY, Wei YQ, et al. Ginsenoside Re attenuates diabetes-associated cognitive deficits in rats. Pharmacol Biochem Behav (2012) 101:93-8. doi:10.1016/j.pbb.2011.12.003

26. Liu J, Yan X, Li L, Zhu Y, Qin K, Zhou L, et al. Ginsennoside Rd attenuates cognitive dysfunction in a rat model of Alzheimer's disease. Neurochem Res (2012) 37:2738-47. doi:10.1007/s11064-012-0866-2

27. Cheng Y, Shen LH, Zhang JT. Anti-amnestic and anti-aging effects of ginsenoside Rgl and Rbl and its mechanism of action. Acta Pharmacol Sin (2005) 26:143-9. doi:10.1111/j.1745-7254.2005.00034.x

28. Lin T, Liu Y, Shi M, Liu X, Li L, Zhao G. Promotive effect of ginsenoside Rd on proliferation of neural stem cells in vivo and in vitro. J Ethnopharmacol (2012) 142:754-61. doi:10.1016/j.jep.2012.05.057

29. Liu Z, Chen J, Huang W, Zeng Z, Yang Y, Zhu B. Ginsenoside Rbl protects rat retinal ganglion cells against hypoxia and oxidative stress. Mol Med Rep (2013) 8:1397-403. doi:10.3892/mmr.2013.1658

30. Qian YH, Han H, Hu XD, Shi LL. Protective effect of ginsenoside Rb1 on betaamyloid protein(1-42)-induced neurotoxicity in cortical neurons. Neurol Res (2009) 31:663-7. doi:10.1179/174313209X385572

31. Wang P, Wei X, Zhang F, Yang K, Qu C, Luo H, et al. Ginsenoside Rg1 of Panax ginseng stimulates the proliferation, odontogenic/osteogenic differentiation and gene expression profiles of human dental pulp stem cells. Phytomedicine (2014) 21:177-83. doi:10.1016/j.phymed.2013.08.021

32. Wu J, Pan Z, Cheng M, Shen Y, Yu H, Wang Q, et al. Ginsenoside Rg1 facilitates neural differentiation of mouse embryonic stem cells via GR-dependent signaling pathway. Neurochem Int (2013) 62:92-102. doi:10.1016/j.neuint.2012. 09.016

33. Xie X, Wang HT, Li CL, Gao XH, Ding JL, Zhao HH, et al. Ginsenoside Rbl protects PC12 cells against beta-amyloid-induced cell injury. Mol Med Rep (2010) 3:635-9. doi:10.3892/mmr_00000308

34. Ma LY, Wang CL, Zhang Q, Du LJ, Chen JM, Xiao PG. Effects of PNS on cerebral blood supply and energy metabolism in mice. Chin Pharmacol Bull (1998) 14:27-9.

35. Gu P, Zhang Y. Effect of Sanqi PNS on Bcl-2 expression and neural cell apoptosis in rats with intracerebral hemorrhage. J Chin Clin Med (2006) 13:527-9.

36. Jia D, Deng Y, Gao J, Liu X, Chu J, Shu Y. Neuroprotective effect of Panax notoginseng plysaccharides against focal cerebral ischemia reperfusion injury in rats. Int J Biol Macromol (2014) 63:177-80. doi:10.1016/j.ijbiomac.2013.10.034

37. Zhou Y, Wang JW, Jiang R, Yao X, Yang B, Cai SZ, et al. [Study on anti-aging effect of ginsenoside Rgl in serial transplantation of hematopoietic stem cells and progenitor cells]. Zhongguo Zhong Yao Za Zhi (2013) 38:2848-53.

38. Chen C, Mu XY, Zhou Y, Shun K, Geng S, Liu J, et al. Ginsenoside Rgl enhances the resistance of hematopoietic stem/progenitor cells to radiation-induced aging in mice. Acta Pharmacol Sin (2014) 35:143-50. doi:10.1038/aps.2013.136

39. Li C, Li Q, Liu YY, Wang MX, Pan CS, Yan L, et al. Protective effects of notoginsenoside R1 on intestinal ischemia-reperfusion injury in rats. Am J Physiol Gastrointest Liver Physiol (2014) 306:G111-22. doi:10.1152/ajpgi.00123.2013

40. Li H, He WY, Lin F, Gou X. Panax notoginseng saponins improve erectile function through attenuation of oxidative stress, restoration of Akt activity and protection of endothelial and smooth muscle cells in diabetic rats with erectile dysfunction. Urol Int (2014) 93(1):92-9. doi:10.1159/000354878

41. Li W, Li P, Liu Z, Du Q, Steinmetz A, Wang N, et al. A Chinese medicine preparation induces neuroprotection by regulating paracrine signaling of brain microvascular endothelial cells. J Ethnopharmacol (2014) 151:686-93. doi:10.1016/j.jep.2013.11.035

42. Lin N, Cai DL, Jin D, Chen Y, Shi JJ. Ginseng panaxoside rb1 reduces body weight in diet-induced obese mice. Cell Biochem Biophys (2014) 68:189-94. doi:10.1007/s12013-013-9688-3

43. Meng X, Sun G, Ye J, Xu H, Wang H, Sun X. Notoginsenoside R1-mediated neuroprotection involves estrogen receptor-dependent crosstalk between Akt and ERK1/2 pathways: a novel mechanism of Nrf2/ARE signaling activation. Free Radic Res (2014) 48:445-60. doi:10.3109/10715762.2014.885117

44. Shen K, Leung SW, Ji L, Huang Y, Hou M, Xu A, et al. Notoginsenoside Ftl activates both glucocorticoid and estrogen receptors to induce endotheliumdependent, nitric oxide-mediated relaxations in rat mesenteric arteries. Biochem Pharmacol (2014) 88(1):66-74. doi:10.1016/j.bcp.2014.01.007

45. Zhang ZG, Niu XY, He XJ, Shu J. Ginsenoside Rg1 reduces toxicity of fine particulate matter on human alveolar epithelial cells: a preliminary observation. Mol Med Rep (2014) 9:989-92. doi:10.3892/mmr.2013.1870

46. Zhou Q, Jiang L, Xu C, Luo D, Zeng C, Liu P, et al. Ginsenoside Rg1 inhibits platelet activation and arterial thrombosis. Thromb Res (2014) 133:57-65. doi:10.1016/j.thromres.2013.10.032

47. Gao B, Shi HL, Li X, Qiu SP, Wu H, Zhang BB, et al. p38 MAPK and ERK1/2 pathways are involved in the pro-apoptotic effect of notoginsenoside Ft1 on human neuroblastoma SH-SY5Y cells. Life Sci (2014) 108:63-70. doi:10.1016/j.lfs.2014.05.010

48. Cui HM, Zhang CG, Lin H, Lu WL, Cheng HP, Wang J. [Determination of effective components in different positions of Panax notoginseng by HPLC]. Zhong Yao Cai (2009) 32:1810-3.

49. Anderson CS, Huang Y, Arima H, Heeley E, Skulina C, Parsons MW, et al. Effects of early intensive blood pressure-lowering treatment on the growth of hematoma and perihematomal edema in acute intracerebral hemorrhage: the Intensive Blood Pressure Reduction in Acute Cerebral Haemorrhage Trial (INTERACT). Stroke (2010) 41:307-12. doi:10.1161/STROKEAHA.109. 561795

50. Chen G, Yang M, Lu Z, Zhang J, Huang H, Liang Y, et al. Microbial transformation of 20(S)-protopanaxatriol-type saponins by Absidia coerulea. J Nat Prod (2007) 70:1203-6. doi:10.1021/np070053v

51. Abou-Chebl A, Reginelli J, Bajzer CT, Yadav JS. Intensive treatment of hypertension decreases the risk of hyperperfusion and intracerebral hemorrhage following carotid artery stenting. Catheter Cardiovasc Interv (2007) 69:690-6. doi:10.1002/ccd.20693

52. Aung HH, Mehendale SR, Wang CZ, Xie JT, McEntee E, Yuan CS. Cisplatin's tumoricidal effect on human breast carcinoma MCF-7 cells was not attenuated by American ginseng. Cancer Chemother Pharmacol (2007) 59:369-74. doi:10.1007/s00280-006-0278-6

53. Chen W, Hu GL, Wang YR, Wang XR. [Determination of six ginsenosides in Panax species by high performance liquid chromatography]. Se Pu (2000) 18:439-41.

54. Yoshikawa M, Morikawa T, Yashiro K, Murakami T, Matsuda H. Bioactive saponins and glycosides. XIX. Notoginseng (3): immunological adjuvant activity of notoginsenosides and related saponins: structures of notoginsenosides-L, $-\mathrm{M}$, and $-\mathrm{N}$ from the roots of Panax notoginseng (Burk.) F. H. Chen. Chem Pharm Bull (Tokyo) (2001) 49:1452-6. doi:10.1248/cpb.49.1452

55. Yoshikawa M, Murakami T, Ueno T, Yashiro K, Hirokawa N, Murakami N, et al. Bioactive saponins and glycosides. VIII. Notoginseng (1): new dammaranetype triterpene oligoglycosides, notoginsenosides-A, $-\mathrm{B},-\mathrm{C}$, and $-\mathrm{D}$, from the dried root of Panax notoginseng (Burk.) F.H. Chen. Chem Pharm Bull (Tokyo) (1997) 45:1039-45. doi:10.1248/cpb.45.1039

56. Adu-Bonsaffoh K, Samuel OA, Binlinla G. Maternal deaths attributable to hypertensive disorders in a tertiary hospital in Ghana. Int J Gynaecol Obstet (2013) 123:110-3. doi:10.1016/j.ijgo.2013.05.017

57. Cui X, Trinh K, Wang YJ. Chinese herbal medicine for chronic neck pain due to cervical degenerative disc disease. Cochrane Database Syst Rev (2010) (1):CD006556. doi:10.1002/14651858.CD006556.pub2

58. Chan EC, Yap SL, Lau AJ, Leow PC, Toh DF, Koh HL. Ultra-performance liquid chromatography/time-of-flight mass spectrometry based metabolomics of 
raw and steamed Panax notoginseng. Rapid Commun Mass Spectrom (2007) 21:519-28. doi:10.1002/rcm.2864

59. Auriat A, Plahta WC, McGie SC, Yan R, Colbourne F. 17beta-Estradiol pretreatment reduces bleeding and brain injury after intracerebral hemorrhagic stroke in male rats. J Cereb Blood Flow Metab (2005) 25:247-56. doi:10.1038/sj.jcbfm.9600026

60. Abou-Chebl A. Endovascular treatment of acute ischemic stroke may be safely performed with no time window limit in appropriately selected patients. Stroke (2010) 41:1996-2000. doi:10.1161/STROKEAHA.110.578997

61. Liu H, Yang J, Du F, Gao X, Ma X, Huang Y, et al. Absorption and disposition of ginsenosides after oral administration of Panax notoginseng extract to rats. Drug Metab Dispos (2009) 37:2290-8. doi:10.1124/dmd.109.029819

62. Tan ZY, Xiong WN, Huang XZ, Liang JQ. [Pharmacokinetics and bioavailability of ginsenoside Rg1 in rats]. Zhong Yao Cai (2013) 36:1121-3.

63. Li X, Sun J, Wang G, Hao H, Liang Y, Zheng Y, et al. Simultaneous determination of panax notoginsenoside $\mathrm{R} 1$, ginsenoside $\mathrm{Rg} 1, \mathrm{Rd}, \mathrm{Re}$ and $\mathrm{Rb} 1$ in rat plasma by HPLC/ESI/MS: platform for the pharmacokinetic evaluation of total panax notoginsenoside, a typical kind of multiple constituent traditional Chinese medicine. Biomed Chromatogr (2007) 21:735-46. doi:10.1002/bmc.813

64. Li L, Sheng Y, Zhang J, Wang C, Guo D. HPLC determination of four active saponins from Panax notoginseng in rat serum and its application to pharmacokinetic studies. Biomed Chromatogr (2004) 18:849-56. doi:10.1002/ bmc. 400

65. Li X, Wang G, Sun J, Hao H, Xiong Y, Yan B, et al. Pharmacokinetic and absolute bioavailability study of total panax notoginsenoside, a typical multiple constituent traditional chinese medicine (TCM) in rats. Biol Pharm Bull (2007) 30:847-51. doi:10.1248/bpb.30.847

66. Xu QF, Fang XL, Chen DF. Pharmacokinetics and bioavailability of ginsenoside Rb1 and Rg1 from Panax notoginseng in rats. J Ethnopharmacol (2003) 84:187-92. doi:10.1016/S0378-8741(02)00317-3

67. Lin L, Liu JX, Zhang Y, Duan CL. Pharmacokinetic studies of ginsenoside Rg1, Re, Rbl and Rd in rats by LC-MS/MS method. Chin Pharm (2009) 44:373-7.

68. CNS TtCNCoN. Criteria for diagnosis of various types of cerebrovascular disease. Chin J Neurol Sci (1996) 29:379.

69. Yuan Y, Zeng X, Luo Y, Li Z, Wu T. Chuanxiong-type preparations for acute ischemic stroke. Cochrane Database Syst Rev (2008) (4):CD005569. doi:10.1002/14651858.CD005569.pub2

70. Jadad AR, Moore RA, Carroll D, Jenkinson C, Reynolds DJ, Gavaghan DJ, et al. Assessing the quality of reports of randomized clinical trials: is blinding necessary? Control Clin Trials (1996) 17:1-12. doi:10.1016/0197-2456(95)00134-4

71. Zhang Y, Yuan F, Zhang X. Clinical study of 130 cases of Lu Lu Tong injection treatment for brain edema after intracerebral hemorrhage (ICH). JPMT (2007) 14:1877-8.

72. Xu YQ, Dong YH. Clinical application of Luo Tai for patients with intracerebral hemorrhage during the phase of hemorrhage absorption. Inner Mongol J Tradit Chin Med (2003) 22:41.

73. Li Q, Yang XY. Observation on the effect of Panax notoginseng saponin for intracerebral hemorrhage in the convalescence phase. Chin J Misdiagn (2004) 4:262-3.

74. Tian ZX, Wang HB, Wei YM. Xue Sai Tong injection applied for the treatment of 36 cases with acute cerebral hemorrhage. J Pract Tradit Chin Intern Med (2004) 18:68

75. Li H, Sun HH. Clinical observation of cerebral hemorrhage treated by Xuesaitong injection. Chin J Integr Tradit West Med Intensive Crit Care (2004) 11:50-2.

76. Xie GQ, Liu JL, Wang XY, Gao C. Clinical observation of thrombus clear to cerebral hemorrhage. Chin Jintegr Med Cardio/Cerebrovasc Dis (2005) 3:496-7.

77. Chen SL, Liang YY, Wang YX. Twenty tow cases of intracerebral hemorrhage in acute phase treated with Xue Sai Tong. Chin J Integr Med Cardio/Cerebrovasc Dis (2006) 4:80-1.

78. Dong YH, Wang XR. The clinical effect of Xue Sai Tong in the treatment of hematoma absorption. J Med Theory Pract (2006) 19:253-4.

79. Zhou YH, Zhang SX, Liu JJ. Observation on the effect of Panax notoginseng saponin for intracerebral hemorrhage in acute phase. Chin J Integr Med Cardio/Cerebrovasc Dis (2007) 5:69-70.

80. Zheng XD. Xue Shuan Tong injection used for twenty-two cases with intracerebral hemorrhage. J Mod Clin Med (2007) 33:112.

81. Tang YH, Guo WL, Rao P, Weng TM, Zhou L. Clinical observations on the effects of herbal preparation with the function of promoting blood circulation and removing blood stasis at the acute and sub-acute phases of primary intracerebral hemorrhage. Mod J Integr Tradit Chin West Med (2007) 16:4285-6.

82. Song Y. Observation on the effect of Xue Shuan Tong for hypertensive intracerebral hemorrhage with small or medium volume. Chin J Misdiagn (2008) 8:8875-6.

83. Ding HY, Dong Q, Han X, Shi LF, Lv CZ. Effects of total Panax notoginseng saponins on $\mathrm{rCBF}$ and neurological function in patients with acute basal ganglia hemorrhage. Neural Inj Funct Reconstr (2008) 3:386-98.

84. Yuan KZ, Zhang F, He HJ. The analysis of the clinical effect of Xue Sai Tong applied for the treatment of patients in the early stage of intracerebral hemorrhage. Chin Community Doctors (2010) 12:136-7.

85. Ding HY, Geng WX. Clinical research on Xue Shuan Tong for the treatment of small volume hypertensive intracerebral hemorrhage in acute phase. China Mod Med (2010) 17:56-9.

86. Gao Y, Dan S, Zhang J, Zhang J, Fan SS, Wang Z. Effects of total Panax notoginseng saponins on neurological function and serum complement C3 in patients with acute intracerebral hemorrhage. Chin Tradit Patent Med (2011) 33:1851-3.

87. Chen KZ. Observation on clinical effect of Panax notoginseng saponin for the treatment of sixty-one patients with acute intracerebral hemorrhage. Hunan J Tradit Chin Med (2013) 29:41-2.

88. Li JM, Gong NX, Li SG, Hu B, Zhou JQ, Guo YH, et al. Observation on Panax Notoginseng's clinical effect of different administrating time for patients with intracerebral hemorrhage. Chin J Integr Tradit West Med Intens Crit Care (2003) 23:546-7.

89. He D, Liu QR, Zhao J, Dong Q, Zhang RL, Han X. Therapeutic efficacy of Xueshuantong on patients with early stage acute intracerebral hemorrhage. Chin J Integr Tradit West Med Intens Crit Care (2002) 9:27-9.

90. Guo XF, Yan YB, Zhao YH, Sun CX, Tan YM, Zhao Q, et al. Clinical research on therapy of invigorating the circulation of blood for the treatment of intracerebral hemorrhage in early stage. Chin J Misdiagn (2002) 2:1355-6.

91. Ogata T, Yasaka M, Wakugawa Y, Inoue T, Ibayashi S, Okada Y. Deep venous thrombosis after acute intracerebral hemorrhage. J Neurol Sci (2008) 272:83-6. doi:10.1016/j.jns.2008.04.032

92. Tveiten A, Ljøstad U, Mygland $\AA$, Naess H. Functioning of long-term survivors of first-ever intracerebral hemorrhage. Acta Neurol Scand (2014) 129(4):269-75. doi:10.1111/ane.12185

93. Frutos Bernal E, Rubio Gil FJ, Martin Corral JC, Marcos Prieto LA, Gonzalez Robledo J. [Prognostic factors in severe traumatic brain injury]. Med Intensiva (2013) 37:327-32. doi:10.1016/j.medin.2012.05.015

94. Chen XF, Song XG. Influence and applied time of $P$. notoginseng on cerebral edema, hematoma volume and $\mathrm{Bcl}-2$ expression in cerebral tissue in rats with acute cerebral hemorrhage. J Clin Neuro (Chinese) (2011) 24:191-3.

95. Zhao XS, Chen ZG, Xu ZF. Effect of early use of Panax notoginseng saponins on inflammation in rats with cerebral hemorrhage. Int Tradit Chin Med (2013) 8:787-9.

96. Xu DY, Huang HB. Analysis of the adverse reactions induced by Sanqi and its preparations. Zhongguo Zhong Yao Za Zhi (2005) 30:1465-8.

97. Ning MH, Jiang F, Xu LP. Thirty cases of Xuesaitong injection treatment of acute intracerebral hemorrhage. J Tradit Chin Med (2004) 8:592-4.

98. Cadilhac DA, Dewey HM, Vos T, Carter R, Thrift AG. The health loss from ischemic stroke and intracerebral hemorrhage: evidence from the North East Melbourne Stroke Incidence Study (NEMESIS). Health Qual Life Outcomes (2010) 8:49. doi:10.1186/1477-7525-8-49

99. Revel-Vilk S, Golomb MR, Achonu C, Stain AM, Armstrong D, Barnes MA, et al. Effect of intracranial bleeds on the health and quality of life of boys with hemophilia. J Pediatr (2004) 144:490-5. doi:10.1016/j.jpeds.2003.12.016

100. Lau J, Ioannidis JP, Terrin N, Schmid CH, Olkin I. The case of the misleading funnel plot. BMJ (2006) 333:597-600. doi:10.1136/bmj.333.7568.597

101. Tang JL, Liu JL. Misleading funnel plot for detection of bias in meta-analysis. J Clin Epidemiol (2000) 53:477-84. doi:10.1016/S0895-4356(99)00204-8

102. Davis SM, Broderick J, Hennerici M, Brun NC, Diringer MN, Mayer SA, et al. Hematoma growth is a determinant of mortality and poor outcome after intracerebral hemorrhage. Neurology (2006) 66:1175-81. doi:10.1212/01.wnl. 0000208408.98482 .99

103. Gao L, Zhao H, Liu Q, Song J, Xu C, Liu P, et al. Improvement of hematoma absorption and neurological function in patients with acute intracerebral hemorrhage treated with Xueshuantong. J Neurol Sci (2012) 323:236-40. doi:10.1016/j.jns.2012.09.028 
104. Luo PD, Luo Y. Clinical study of notoginsenosiole therapy for cerebral hemorrhage. J Prev Med (2008) 35:4307-9.

105. Leys D, Bodenant M, Cordonnier C. [Intra-cerebral haemorrhages in the elderly]. Rev Prat (2012) 62:1239-42.

106. Fujii Y, Takeuchi S, Tanaka R, Koike T, Sasaki O, Minakawa T. Liver dysfunction in spontaneous intracerebral hemorrhage. Neurosurgery (1994) 35:592-6. doi:10.1097/00006123-199410000-00003

107. Immonen RJ, Kharatishvili I, Grohn H, Pitkanen A, Grohn OH. Quantitative MRI predicts long-term structural and functional outcome after experimental traumatic brain injury. Neuroimage (2009) 45:1-9. doi:10.1016/j.neuroimage. 2008.11.022

108. Zhao G, Wang X. The hemostatic component of Panax notoginseng: dencichine. Chin Tradit Herb Drugs (1986) 17:34-6.

109. Huang LF, Shi HL, Gao B, Wu H, Yang L, Wu XJ, et al. Decichine enhances hemostasis of activated platelets via AMPA receptors. Thromb Res (2014) 133:848-54. doi:10.1016/j.thromres.2014.02.009

110. Chan CM, Zilberberg MD. Preferences in traumatic intracranial hemorrhage: bleeding vs. clotting. Crit Care (2010) 14:153. doi:10.1186/cc8996

111. Gebel JM, Brott TG, Sila CA, Tomsick TA, Jauch E, Salisbury S, et al. Decreased perihematomal edema in thrombolysis-related intracerebral hemorrhage compared with spontaneous intracerebral hemorrhage. Stroke (2000) 31:596-600. doi:10.1161/01.STR.31.3.596

112. Lauer A, Schlunk F, Van Cott EM, Steinmetz H, Lo EH, Foerch C. Antiplatelet pretreatment does not increase hematoma volume in experimental intracerebral hemorrhage. J Cereb Blood Flow Metab (2011) 31:1736-42. doi:10.1038/ jcbfm.2011.22

113. Lok J, Leung W, Murphy S, Butler W, Noviski N, Lo EH. Intracranial hemorrhage: mechanisms of secondary brain injury. Acta Neurochir Suppl (2011) 111:63-9. doi:10.1007/978-3-7091-0693-8_11

114. Chen G, Mu L, Zhang X, Hou S, Nan H. [In vivo distribution and pharmacokinetics of multiple effective components contained in Panax notoginseng saponins after intratympanic administration]. Zhongguo Zhong Yao Za Zhi (2011) 36:1815-20.

115. Emiru T, Bershad EM, Zantek ND, Datta YH, Rao GH, Hartley EW, et al. Intracerebral hemorrhage: a review of coagulation function. Clin Appl Thromb Hemost (2013) 19:652-62. doi:10.1177/1076029612454938

116. Allard CB. Retraction note to: ginsenoside-Rg1 enhances angiogenesis and ameliorates ventricular remodeling in a rat model of myocardial infarction. $J$ Mol Med (Berl) (2013) 91:645.

117. Allard CB, Scarpelini S, Rhind SG, Baker AJ, Shek PN, Tien H, et al. Abnormal coagulation tests are associated with progression of traumatic intracranial hemorrhage. J Trauma (2009) 67:959-67. doi:10.1097/TA.0b013e3181ad5d37

118. Chai H, Dong Y, Wang X, Zhou W. Ginsenoside Rb1 attenuates homocysteineaugmented guidewire injury-induced intimal hyperplasia in mice. J Surg Res (2009) 157:193-8. doi:10.1016/j.jss.2008.07.005

119. Xu HL, Liu WB, Rao MR. [Effect of sanchinoside Rg1 on experimental thrombosis and its mechanisms]. Yao Xue Xue Bao (1997) 32:502-5.

120. Liu DH, Chen YM, Liu Y, Hao BS, Zhou B, Wu L, et al. Ginsenoside Rb1 reverses $\mathrm{H} 2 \mathrm{O} 2$-induced senescence in human umbilical endothelial cells: involvement of eNOS pathway. J Cardiovasc Pharmacol (2012) 59:222-30. doi:10.1097/FJC.0b013e31823c1d34

121. Xia R, Zhao B, Wu Y, Hou JB, Zhang L, Xu JJ, et al. Ginsenoside $\mathrm{Rb} 1$ preconditioning enhances eNOS expression and attenuates myocardial ischemia/reperfusion injury in diabetic rats. J Biomed Biotechnol (2011) 2011:767930. doi:10.1155/2011/767930

122. Chen HZ, Li YF, Zhong JH, Fan XH. [Identification of major components of traditional Chinese medicine Naodesheng tablet by HPLC-DAD-MS(n)]. Zhejiang Da Xue Xue Bao Yi Xue Ban (2012) 41:32-42.

123. Berek L, Szabo D, Petri IB, Shoyama Y, Lin YH, Molnar J. Effects of naturally occurring glucosides, solasodine glucosides, ginsenosides and parishin derivatives on multidrug resistance of lymphoma cells and leukocyte functions. In vivo (2001) 15:151-6.

124. Garrett MC, Otten ML, Starke RM, Komotar RJ, Magotti P, Lambris JD, et al. Synergistic neuroprotective effects of C3a and C5a receptor blockade following intracerebral hemorrhage. Brain Res (2009) 1298:171-7. doi:10.1016/j. brainres.2009.04.047

125. Chang Y, Lai PH, Wang CC, Chen SC, Chang WC, Sung HW. Mesothelium regeneration on acellular bovine pericardia loaded with an angiogenic agent (ginsenoside Rg1) successfully reduces postsurgical pericardial adhesions. $J$ Thorac Cardiovasc Surg (2006) 132:867-74. doi:10.1016/j.jtcvs.2006.06.029

126. Gong Y, Xi G, Wan S, Gu Y, Keep RF, Hua Y. Effects of aging on complement activation and neutrophil infiltration after intracerebral hemorrhage. Acta Neurochir Suppl (2008) 105:67-70. doi:10.1007/978-3-211-09469-3_14

127. Loftspring MC, McDole J, Lu A, Clark JF, Johnson AJ. Intracerebral hemorrhage leads to infiltration of several leukocyte populations with concomitant pathophysiological changes. J Cereb Blood Flow Metab (2009) 29:137-43. doi:10.1038/jcbfm.2008.114

128. Yao XH, Li XJ. [Protective effects and its mechanism of panaxatriol saponins isolated from Panax notoginseng on cerebral ischemia]. Zhongguo Zhong Yao Za Zhi (2002) 27:371-3.

129. Lu T, Jiang Y, Zhou Z, Yue X, Wei N, Chen Z, et al. Intranasal ginsenoside Rb1 targets the brain and ameliorates cerebral ischemia/reperfusion injury in rats. Biol Pharm Bull (2011) 34:1319-24. doi:10.1248/bpb.34.1319

130. Shi Y, Han B, Yu X, Qu S, Sui D. Ginsenoside Rb3 ameliorates myocardial ischemia-reperfusion injury in rats. Pharm Biol (2011) 49:900-6. doi:10.3109/ 13880209.2011 .554845

131. Gong W, Xiao Y, Zhang M, Wang Y. [Synergistic protective effects of salvianolic acids and Panax notoginseng saponins on cardiomyocytes with hypoxiareoxygenation injury]. Zhongguo Zhong Yao Za Zhi (2013) 38:1046-51.

132. Sun Q, Meng QT, Jiang Y, Liu HM, Lei SQ, Su WT, et al. Protective effect of ginsenoside $\mathrm{Rb} 1$ against intestinal ischemia-reperfusion induced acute renal injury in mice. PLoS One (2013) 8:e80859. doi:10.1371/journal.pone. 0080859

133. Lan TH, Xu ZW, Wang Z, Wu YL, Wu WK, Tan HM. Ginsenoside Rb1 prevents homocysteine-induced endothelial dysfunction via PI3K/Akt activation and PKC inhibition. Biochem Pharmacol (2011) 82:148-55. doi:10.1016/j.bcp. 2011.04.001

134. Cheung LW, Leung KW, Wong CK, Wong RN, Wong AS. Ginsenoside-Rg1 induces angiogenesis via non-genomic crosstalk of glucocorticoid receptor and fibroblast growth factor receptor-1. Cardiovasc Res (2011) 89:419-25. doi:10.1093/cvr/cvq300

135. Leung KW, Ng HM, Tang MK, Wong CC, Wong RN, Wong AS. Ginsenoside-Rg1 mediates a hypoxia-independent upregulation of hypoxia-inducible factorlalpha to promote angiogenesis. Angiogenesis (2011) 14:515-22. doi:10.1007/ s10456-011-9235-Z

136. Tang B, Qu Y, Wang D, Mu D. Targeting hypoxia inducible factor-1alpha: a novel mechanism of ginsenoside Rg1 for brain repair after hypoxia/ischemia brain damage. CNS Neurol Disord Drug Targets (2011) 10:235-8. doi:10.2174/ 187152711794480456

137. Wang DJ, Li QY, Xu SJ, Zeng N. [Effect of ginsenoside Rg1 on angiogenesis after neonatal hypoxia ischemia brain damage in rats]. Sichuan Da Xue Xue Bao Yi Xue Ban (2011) 42:503-7.

138. Yang N, Chen P, Tao Z, Zhou N, Gong X, Xu Z, et al. Beneficial effects of ginsenoside-Rg1 on ischemia-induced angiogenesis in diabetic mice. Acta Biochim Biophys Sin (Shanghai) (2012) 44:999-1005. doi:10.1093/abbs/gms092

139. Shen K, Ji L, Gong C, Ma Y, Yang L, Fan Y, et al. Notoginsenoside Ft1 promotes angiogenesis via HIF-1alpha mediated VEGF secretion and the regulation of PI3K/AKT and Raf/MEK/ERK signaling pathways. Biochem Pharmacol (2012) 84:784-92. doi:10.1016/j.bcp.2012.05.024

140. Zheng H, Liu C, Ou Y, Zhang Y, Fu X. Total saponins of Panax notoginseng enhance VEGF and relative receptors signals and promote angiogenesis derived from rat bone marrow mesenchymal stem cells. J Ethnopharmacol (2013) 147:595-602. doi:10.1016/j.jep.2013.03.043

141. Chen S, Liu J, Liu X, Fu Y, Zhang M, Lin Q, et al. Panax notoginseng saponins inhibit ischemia-induced apoptosis by activating PI3K/Akt pathway in cardiomyocytes. J Ethnopharmacol (2011) 137:263-70. doi:10.1016/j.jep. 2011.05.011

142. Ma J, Li W, Tian R, Lei W. Ginsenoside Rg1 promotes peripheral nerve regeneration in rat model of nerve crush injury. Neurosci Lett (2010) 478:66-71. doi:10.1016/j.neulet.2010.04.064

143. He W, Wu WK, Wu YL, Yang XH, Lin QX, Yu WH. Ginsenoside-Rg1 mediates microenvironment-dependent endothelial differentiation of human mesenchymal stem cells in vitro. JAsian Nat Prod Res (2011) 13:1-11. doi:10.1080/ 10286020.2010.535519

144. Arauz A, Berge E, Sandercock P. Third International Stroke Trial 3: an update. Curr Opin Neurol (2014) 27:8-12. doi:10.1097/WCO.0000000000000045 
145. Balsevich JJ, Bishop GG, Deibert LK. Use of digitoxin and digoxin as internal standards in HPLC analysis of triterpene saponin-containing extracts. Phytochem Anal (2009) 20:38-49. doi:10.1002/pca.1095

146. Chang SH, Choi Y, Park JA, Jung DS, Shin J, Yang JH, et al. Anti-inflammatory effects of BT-201, an $n$-butanol extract of Panax notoginseng, observed in vitro and in a collagen-induced arthritis model. Clin Nutr (2007) 26:785-91. doi:10.1016/j.clnu.2007.07.008

147. Luo FC, Wang SD, Li K, Nakamura H, Yodoi J, Bai J. Panaxatriol saponins extracted from Panax notoginseng induces thioredoxin-1 and prevents 1methyl-4-phenylpyridinium ion-induced neurotoxicity. J Ethnopharmacol (2010) 127:419-23. doi:10.1016/j.jep.2009.10.023

148. Na JY, Kim S, Song K, Lim KH, Shin GW, Kim JH, et al. Anti-apoptotic activity of ginsenoside Rb1 in hydrogen peroxide-treated chondrocytes: stabilization of mitochondria and the inhibition of caspase-3. J Ginseng Res (2012) 36:242-7. doi:10.5142/jgr.2012.36.3.242

149. Hashimoto R, Yu J, Koizumi H, Ouchi Y, Okabe T. Ginsenoside Rbl prevents $\mathrm{MPP}(+)$-induced apoptosis in PC12 cells by stimulating estrogen receptors with consequent activation of ERK1/2, Akt and inhibition of SAPK/JNK, p38 MAPK. Evid Based Complement Alternat Med (2012) 2012:693717. doi:10.1155/2012/693717

150. Xie XS, Liu HC, Yang M, Zuo C, Deng Y, Fan JM. Ginsenoside Rb1, a panoxadiol saponin against oxidative damage and renal interstitial fibrosis in rats with unilateral ureteral obstruction. Chin J Integr Med (2009) 15:133-40. doi:10.1007/s11655-009-0133-9

151. Ye R, Kong X, Yang Q, Zhang Y, Han J, Li P, et al. Ginsenoside Rd in experimental stroke: superior neuroprotective efficacy with a wide therapeutic window. Neurotherapeutics (2011) 8:515-25. doi:10.1007/s13311-011-0051-3

152. Ye R, Kong X, Yang Q, Zhang Y, Han J, Zhao G. Ginsenoside Rd attenuates redox imbalance and improves stroke outcome after focal cerebral ischemia in aged mice. Neuropharmacology (2011) 61:815-24. doi:10.1016/j.neuropharm.2011. 05.029

153. Ye R, Yang Q, Kong X, Han J, Zhang X, Zhang Y, et al. Ginsenoside Rd attenuates early oxidative damage and sequential inflammatory response after transient focal ischemia in rats. Neurochem Int (2011) 58:391-8. doi:10.1016/j.neuint. 2010.12.015

154. Li XY, Liang J, Tang YB, Zhou JG, Guan YY. Ginsenoside Rd prevents glutamateinduced apoptosis in rat cortical neurons. Clin Exp Pharmacol Physiol (2010) 37:199-204. doi:10.1111/j.1440-1681.2009.05286.x

155. Zhang X, Shi M, Bjørås M, Wang W, Zhang G, Han J, et al. Ginsenoside Rd promotes glutamate clearance by up-regulating glial glutamate transporter GLT-1 via PI3K/AKT and ERK1/2 pathways. Front Pharmacol (2013) 4:152. doi:10.3389/fphar.2013.00152

156. Zhang YX, Wang L, Xiao EL, Li SJ, Chen JJ, Gao B, et al. Ginsenoside-Rd exhibits anti-inflammatory activities through elevation of antioxidant enzyme activities and inhibition of JNK and ERK activation in vivo. Int Immunopharmacol (2013) 17:1094-100. doi:10.1016/j.intimp.2013.10.013

157. Liu Q, Kou JP, Yu BY. Ginsenoside Rg1 protects against hydrogen peroxideinduced cell death in PC12 cells via inhibiting NF-kappaB activation. Neurochem Int (2011) 58:119-25. doi:10.1016/j.neuint.2010.11.004

158. Wang J, Xu HM, Yang HD, Du XX, Jiang H, Xie JX. Rg1 reduces nigral iron levels of MPTP-treated C57BL6 mice by regulating certain iron transport proteins. Neurochem Int (2009) 54:43-8. doi:10.1016/j.neuint.2008.10.003

159. Yan J, Liu Q, Dou Y, Hsieh Y, Liu Y, Tao R, et al. Activating glucocorticoid receptor-ERK signaling pathway contributes to ginsenoside $\mathrm{Rg} 1$ protection against beta-amyloid peptide-induced human endothelial cells apoptosis. $J$ Ethnopharmacol (2013) 147:456-66. doi:10.1016/j.jep.2013.03.039

160. Yu SH, Huang HY, Korivi M, Hsu MF, Huang CY, Hou CW, et al. Oral Rg1 supplementation strengthens antioxidant defense system against exercise-induced oxidative stress in rat skeletal muscles. J Int Soc Sports Nutr (2012) 9:23. doi:10.1186/1550-2783-9-23

161. Korivi M, Hou CW, Huang CY, Lee SD, Hsu MF, Yu SH, et al. Ginsenoside-Rg1 protects the liver against exhaustive exercise-induced oxidative stress in rats. Evid Based Complement Alternat Med (2012) 2012:932165. doi:10.1155/2012/ 932165

162. Cheng W, Wu D, Zuo Q, Wang Z, Fan W. Ginsenoside Rb1 prevents interleukin1 beta induced inflammation and apoptosis in human articular chondrocytes. Int Orthop (2013) 37:2065-70. doi:10.1007/s00264-013-1990-6

163. Kim DH, Chung JH, Yoon JS, Ha YM, Bae S, Lee EK, et al. Ginsenoside Rd inhibits the expressions of iNOS and COX-2 by suppressing NF-kappaB in
LPS-stimulated RAW264.7 cells and mouse liver. J Ginseng Res (2013) 37:54-63. doi:10.5142/jgr.2013.37.54

164. Lin WM, Zhang YM, Moldzio R, Rausch WD. Ginsenoside Rd attenuates neuroinflammation of dopaminergic cells in culture. J Neural Transm Suppl (2007):105-12. doi:10.1007/978-3-211-73574-9_13

165. Lee IA, Hyam SR, Jang SE, Han MJ, Kim DH. Ginsenoside Re ameliorates inflammation by inhibiting the binding of lipopolysaccharide to TLR4 on macrophages. J Agric Food Chem (2012) 60:9595-602. doi:10.1021/ jf301372g

166. Zou Y, Tao T, Tian Y, Zhu J, Cao L, Deng X, et al. Ginsenoside Rgl improves survival in a murine model of polymicrobial sepsis by suppressing the inflammatory response and apoptosis of lymphocytes. J Surg Res (2013) 183:760-6. doi:10.1016/j.jss.2013.01.068

167. Ma X, Xie X, Zuo C, Fan J. [Effects of ginsenoside Rg1 on streptozocin-induced diabetic nephropathy in rats]. Sheng Wu Yi Xue Gong Cheng Xue Za Zhi (2010) 27:342-7.

168. Liu RY, Wang JJ, Qiu X, Wu JM. Acute hyperglycemia together with hematoma of high-glucose blood exacerbates neurological injury in a rat model of intracerebral hemorrhage. Neurosci Bull (2014) 30:90-8. doi:10.1007/s12264-0131371-6

169. Chiu CD, Chen CC, Shen CC, Chin LT, Ma HI, Chuang HY, et al. Hyperglycemia exacerbates intracerebral hemorrhage via the downregulation of aquaporin-4: temporal assessment with magnetic resonance imaging. Stroke (2013) 44:1682-9. doi:10.1161/STROKEAHA.113.675983

170. Xia W, Sun C, Zhao Y, Wu L. Hypolipidemic and antioxidant activities of sanchi (radix notoginseng) in rats fed with a high fat diet. Phytomedicine (2011) 18:516-20. doi:10.1016/j.phymed.2010.09.007

171. Xiong Y, Shen L, Liu KJ, Tso P, Xiong Y, Wang G, et al. Antiobesity and antihyperglycemic effects of ginsenoside Rb1 in rats. Diabetes (2010) 59:2505-12. doi: $10.2337 / \mathrm{db} 10-0315$

172. Lee KT, Jung TW, Lee HJ, Kim SG, Shin YS, Whang WK. The antidiabetic effect of ginsenoside Rb2 via activation of AMPK. Arch Pharm Res (2011) 34:1201-8. doi:10.1007/s12272-011-0719-6

173. Kim EJ, Lee HI, Chung KJ, Noh YH, Ro Y, Koo JH. The ginsenoside-Rb2 lowers cholesterol and triacylglycerol levels in 3T3-L1 adipocytes cultured under high cholesterol or fatty acids conditions. BMB Rep (2009) 42:194-9. doi:10.5483/BMBRep.2009.42.4.194

174. Han DH, Kim SH, Higashida K, Jung SR, Polonsky KS, Klein S, et al. Ginsenoside Re rapidly reverses insulin resistance in muscles of high-fat diet fed rats. Metabolism (2012) 61:1615-21. doi:10.1016/j.metabol.2012.04.008

175. Lee HM, Lee OH, Kim KJ, Lee BY. Ginsenoside Rg1 promotes glucose uptake through activated AMPK pathway in insulin-resistant muscle cells. Phytother Res (2012) 26:1017-22. doi:10.1002/ptr.3686

176. Yong-Xin X, Jian-Jun Z. Evaluation of anti-fatigue activity of total saponins of Radix notoginseng. Indian J Med Res (2013) 137:151-5. doi:10.4236/wjns. 2014.41002

177. Wang JH, Peng L, Wei C, Luo QZ, Cao XJ. Thirty cases of Xuesetong pill treatment of blood stasis syndrome during stroke recovery. Shaanxi Tradit Chin Med (2007) 28:1314-5.

Conflict of Interest Statement: The authors declare that the research was conducted in the absence of any commercial or financial relationships that could be construed as a potential conflict of interest.

Received: 25 February 2014; accepted: 03 December 2014; published online: 07 January 2015.

Citation: Xu D, Huang P, Yu Z, Xing DH, Ouyang S and Xing G (2015) Efficacy and safety of Panax notoginseng saponin therapy for acute intracerebral hemorrhage, metaanalysis, and mini review of potential mechanisms of action. Front. Neurol. 5:274. doi: 10.3389/fneur.2014.00274

This article was submitted to Neurotrauma, a section of the journal Frontiers in Neurology.

Copyright (C) 2015 Xu, Huang, Yu, Xing, Ouyang and Xing. This is an open-access article distributed under the terms of the Creative Commons Attribution License (CC BY). The use, distribution or reproduction in other forums is permitted, provided the original author(s) or licensor are credited and that the original publication in this journal is cited, in accordance with accepted academic practice. No use, distribution or reproduction is permitted which does not comply with these terms. 